\title{
A novel IMU-based clinical assessment protocol for Axial Spondyloarthritis: A protocol validation study
}

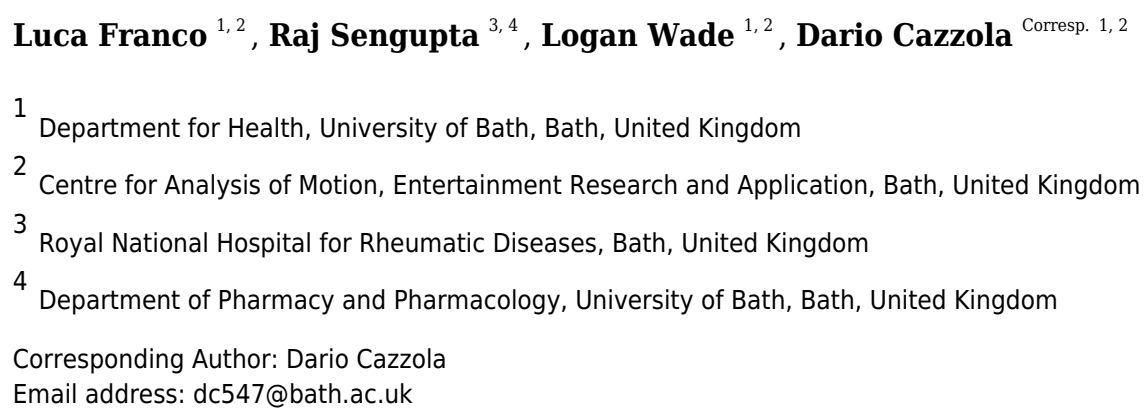

Clinical assessment of spinal impairment in Axial Spondyloarthritis is currently performed using the Bath Ankylosing Spondylitis Metrological Index (BASMI). Despite being appreciated for its simplicity, the BASMI index lacks sensitivity and specificity of spinal changes, demonstrating poor association with radiographical range of motion (ROM). Inertial measurement units (IMUs) have shown promising results as a cost-effective method to quantitatively examine movement of the human body, however errors due to sensor angular drift have limited their application to a clinical space. Therefore, this paper presents a wearable sensor (IMU) protocol that facilitates unrestrained orientation measurements in space while limiting sensor angular drift through a novel constraintbased approach. Eleven healthy male participants performed five BASMI-inspired functional movements where spinal ROM and continuous kinematics were calculated for five spine segments and four spinal joint levels (lumbar, lower thoracic, upper thoracic and cervical). A Bland-Altman analysis was used to assess the level of agreement on range of motion measurements, whilst intraclass correlation coefficient (ICC), standardised error measurement (SEM), and minimum detectable change (MDC) to assess relative and absolute reliability. Continuous kinematics error was investigated through root mean square error (RMSE), maximum absolute error (MAE) and Spearman correlation coefficient $(\rho)$. The overall error in the measurement of continuous kinematic measures was low in both the sagittal $\left(\right.$ RMSE $\left.=2.1^{\circ}\right)$, and frontal plane $\left(R M S E=2.3^{\circ}\right)$. ROM limits of agreement (LoA) and minimum detectable change were excellent for the sagittal plane (maximum value LOA $1.9^{\circ}$ and MDC $2.4^{\circ}$ ) and fair for lateral flexion (overall value LOA $4.8^{\circ}$ and MDC $\left.5.7^{\circ}\right)$. The reliability analysis showed excellent level of agreement (ICC $\left.>0.9\right)$ for both segment and joint ROM across all movements. The results from this study demonstrated better or equivalent accuracy than previous studies and were considered acceptable for 
application in a clinical setting. The protocol has shown to be a valuable tool for the assessment of spinal ROM and kinematics, but a clinical validation study on Axial Spondyloarthritis patients is required for the development and testing of a novel mobility index. 


\section{A novel IMU-based clinical assessment protocol for Axial} Spondyloarthritis: A protocol validation study

Luca Franco ${ }^{1,2}$, Raj Sengupta ${ }^{3,4}$, Logan Wade ${ }^{1,2}$, Dario Cazzola ${ }^{1,2}$

1 University of Bath, Department for Health, Bath, United Kingdom

2 Centre for the Analysis of Motion, Entertainment Research and Application, Bath, United Kingdom

3 University of Bath, Department of Pharmacy and Pharmacology, Bath, United Kingdom

4 Royal National Hospital for Rheumatic Diseases, Bath, United Kingdom

Corresponding Author:

Dr Dario Cazzola

Email address: d.cazzola@bath.ac.uk

\section{Abstract}

Clinical assessment of spinal impairment in Axial Spondyloarthritis is currently performed using the Bath Ankylosing Spondylitis Metrological Index (BASMI). Despite being appreciated for its simplicity, the BASMI index lacks sensitivity and specificity of spinal changes, demonstrating poor association with radiographical range of motion (ROM). Inertial measurement units (IMUs) have shown promising results as a cost-effective method to quantitatively examine movement of the human body, however errors due to sensor angular drift have limited their application to a clinical space. Therefore, this paper presents a wearable sensor (IMU) protocol that facilitates unrestrained orientation measurements in space while limiting sensor angular drift through a novel constraint-based approach. Eleven healthy male participants performed five BASMIinspired functional movements where spinal ROM and continuous kinematics were calculated for five spine segments and four spinal joint levels (lumbar, lower thoracic, upper thoracic and cervical). A Bland-Altman analysis was used to assess the level of agreement on range of motion measurements, whilst intraclass correlation coefficient (ICC), standardised error measurement (SEM), and minimum detectable change (MDC) to assess relative and absolute reliability. 
27 Continuous kinematics error was investigated through root mean square error (RMSE),

28 maximum absolute error (MAE) and Spearman correlation coefficient $(\rho)$. The overall error in the measurement of continuous kinematic measures was low in both the sagittal $\left(\mathrm{RMSE}=2.1^{\circ}\right)$, and frontal plane $\left(\mathrm{RMSE}=2 \cdot 3^{\circ}\right)$. ROM limits of agreement $(\mathrm{LoA})$ and minimum detectable change were excellent for the sagittal plane (maximum value LoA $1.9^{\circ}$ and MDC $2.4^{\circ}$ ) and fair for lateral flexion (overall value $\operatorname{LoA} 4.8^{\circ}$ and $\mathrm{MDC} 5.7^{\circ}$ ). The reliability analysis showed excellent level of agreement (ICC $>0.9$ ) for both segment and joint ROM across all movements. The results from this study demonstrated better or equivalent accuracy than previous studies and were considered acceptable for application in a clinical setting. The protocol has shown to be a valuable tool for the assessment of spinal ROM and kinematics, but a clinical validation study on Axial Spondyloarthritis patients is required for the development and testing of a novel mobility index.

\section{Introduction}

Axial Spondyloarthritis (axSpA), which encompasses Ankylosing Spondylitis (AS) and its nonradiographic form (nr-axSpA) (Baraliakos \& Braun 2015), is an auto-immune disease that presents with chronic inflammation of the spine. Ultimately this results in joint stiffness, entheseal inflammation, and in more severe cases, excessive bone growth and fusion (Akkoc 2018; McGonagle \& Benjamin 2009). These structural changes, in combination with pain, can severely restrict movement and result in functional impairment that often leads to loss of productivity, absence from work (Haglund et al. 2013), job loss (Rafia et al. 2012), difficulty performing everyday tasks (Dagfinrud et al. 2005) and decreased quality of life (Kawalec \& Malinowski 2015). Healthcare and private costs related to AS are substantial worldwide (Akkoc et al. 2015; Kruger et al. 2018; Walsh et al. 2018), with estimated individual patient costs in the UK (including NHS costs, patient costs, and societal costs) of $£ 19,016$ per year (Cooksey et al. 2015). Therefore, methods to assess and treat this disease must be cost effective and accurate to reduce the detriment to both patients and the healthcare system.

Currently, patients with axSpA are clinically assessed through subjective and qualitative patient reported outcomes, such as the Bath Ankylosing Spondylitis Functional Index (BASFI)(Calin et 
al. 1994). Other more objective measures, such as the Bath Ankylosing Spondylitis Metrological Index (BASMI) (Jenkinson et al. 1994), are performed by a clinician and provide an overall measure of a patients' spinal impairment. While the BASMI is widely and reliably used in clinical settings (Calvo-Gutierrez et al. 2016), it lacks specificity in detecting meaningful changes (Martindale et al. 2012) and correlates poorly with spinal range of motion (ROM) during radiographic analysis (Rezvani et al. 2012). These biases are likely due to limited information about movements from individual spinal segments and changes in spinal ROM. While these limitations could be overcome by motion capture (Cappello et al. 2005; Mousavi et al. 2018), or imaging techniques like fluoroscopy (Cox et al. 2001) and dynamic MRI (Kulig et al. 2004), these systems are generally operationally complex and expensive. On the other hand, inertial measurement units (IMUs) offer a low cost, user-friendly alternative that is already widely used in sport (Ahmadi et al. 2010; Nuesch et al. 2017) and clinical applications (Beange et al. 2019).

The primary barrier to clinical applications of IMU movement tracking are magnetic field distortions and angular velocity integration, which results in angular drift (de Vries et al. 2009; Takeda et al. 2009). This is especially prevalent on the vertical axis, although it can be mitigated with the adoption of a tailored Kalman filter or kinematic constraints (Laidig et al. 2017b; Slajpah et al. 2014). To limit such errors when measuring knee angle during walking, Favre et al. (2008) used a fusion algorithm to calculate angular velocity from quaternion-based time integration of gyroscope data, which was then corrected by the accelerometer inclination data. The results were good for flexion/extension and adduction/abduction (offset error less than $3^{\circ}$ ), but internal/external rotation showed offset errors up to $9^{\circ}$. Alternatively, kinematic constraints restrict movement of erroneous axes and were used by Laidig et al. (2017a) to measure elbow joint angles while compensating for magnetic disturbances (flexion/extension average offset $\left.4.1^{\circ}\right)$. Sensor fusion combined with kinematic constraints were also adopted for flexion/extension angles and showed low root mean square errors $\left(3^{\circ}\right)$ during gait (Seel et al. 2014) and low average measurement error (3.4 $)$ during running (Cooper et al. 2009).

A number of studies have examined spinal specific movements using IMUs, however use of multiple sensors was limited (Chhikara et al. 2010; Lee et al. 2011a). Such an approach would provide only a partial picture for a complex disease like axSpA, where inflammation and 
86 functional impairment can have various impacts across the entire spine (Raine \& Keat 2018;

87 Taurog et al. 2016). Garrido-Castro et al. (2018) presented a study adopting IMUs for posture

88 assessment in $\operatorname{axSpA}$, however they only focussed on static measurements of the lumbar and cervical segments using four sensors with some ROM measurements but no kinematics. ArandaValera et al. (2018) used the ViMove IMU system for dynamic spinal assessment and found good correlation with the BASMI (0.60-0.92) but high root mean square error for half of the measurements carried out: cervical rotation $\left(9.4^{\circ}\right)$ and lateral flexion $\left(7.4^{\circ}\right)$, and lumbar lateral flexion (8.3 ${ }^{\circ}$. Mjøsund et al. (2017) also examined the ViMove system and recorded much lower errors for the lumbar spine (flexion RMSE $1.82 \pm 1.00^{\circ}$, and right and left lateral flexion RMSE respectively $0.77 \pm 0.24^{\circ}$ and $\left.0.98 \pm 0.69^{\circ}\right)$. The substantial discrepancy in RMSE between Aranda-Valera et al. (2018) and Mjøsund et al. (2017) studies is grounded on the difference in the way the angles are calculated and the type of gold standard measure used. Aranda adopts the projection of one-dimensional spinal segments - delimited by pairs of reflective markers placed on the body - on anatomical planes (Garrido-Castro et al. 2012), whereas the kinematics analysis Mjøsund et al. (2017) replicates ViMove's angles with marker clusters rigidly attached to them, however they were limited to only examining the lumbar spine.

To improve IMU spinal analysis beyond previous studies, sensor number optimisation, effective kinematic constraints strategies, and a well-designed protocol are likely to be the most reliable methods to accurately measure spinal movement. Therefore this study applied a novel movement-specific kinematic constraints based on the a priori knowledge of the anatomical characteristics of the joints, like degrees of freedom and ROM, and takes advantage of the simplicity of the functional movements, which are substantially planar, by constraining specific sensor axes on global reference planes. This study aims to (i) devise a novel IMU-based protocol for spinal assessment with potential application in axSpA, and (ii) validate the IMU-based protocol against simultaneous measurements taken via an optical motion capture system.

\section{Materials and methods}

\section{Experimental data collection.}

Eleven participants (age $27.3 \pm 5.5$ years, mass $76.1 \pm 7.7 \mathrm{~kg}$, height $1.81 \pm 0.06 \mathrm{~m}$, one of which female) gave written informed consent to take part in this study and ethics were approved by the 
115 Research Ethics Approval Committee for Health [EP 17/18 128] at the University of Bath.

116 Participants were required to be between 18-40 years old and have no history of bone, joint, or

117 neuromuscular problems, or a current musculoskeletal injury.

118 IMUs (Avanti, Delsys inc., Natick (MA), USA) used in this study were equipped with a triaxial 119 accelerometer ( $\pm 2 \mathrm{~g}$ range), a triaxial gyroscope $\left( \pm 2000^{\circ} \mathrm{S}^{-1}\right.$ range $)$, and a triaxial magnetometer.

120 Wireless IMU data was recorded using Delys software (EMGworks Acquisition 4.5.4, Delsys

121 inc., Natick, MA, USA) which included the application of a Delsys proprietary Kalman filter to

122 convert the multi-axial sensor data into a quaternion-based orientation. The sensor data was

123 sampled at $74 \mathrm{~Hz}$ and then downsampled to $50 \mathrm{~Hz}$. IMU position and orientation in 3D space

124 was compared to gold standard motion capture using 12 infrared cameras (Oqus, Qualisys,

125 Göteborg, Sweden) and tracked via custom 3D printed clusters attached to each IMU device

126 (Fig. 1A). Motion capture data was collected using Qualisys Track Manager (Qualisys QTM

127 2019.2, Qualisys AB, Göteborg, Sweden) at a sampling frequency of $100 \mathrm{~Hz}$ and then

128 downsampled to $50 \mathrm{~Hz}$. Both IMU and motion capture data were filtered using a zero-phase low

129 pass $2^{\text {nd }}$ order Butterworth filter with a cut-off frequency of $5 \mathrm{~Hz}$ (Charry et al. 2011). The two

130 systems were triggered simultaneously via a custom triggering system sending a TTL signal

131 from the EMG station to the motion capture system.

132 Sensor locations were based on Lee et al. (2011b) with minor modifications. Each participant

133 was equipped with 5 IMUs: one sensor was attached using double-sided tape directly on the skin

134 (Fig. 1B) at the height of the spinous processes of sacrum one (S1). Three sensors were attached

135 to foam supports (Fig. 1B) that acted to reduce soft tissue artefact by increasing the attachment

136 surface with the skin. These sensors were placed over first lumbar vertebra (L1), first and sixth

137 thoracic vertebra (T1 and T6). Finally, the last sensor was fixed on the head occiput with a head

138 band. The spinous processes were located by palpation and measuring the distance between the

139 spinous processes C7 and S1 with a flexible ruler (Ernst et al. 2013).

FIGURE 1 here

141

142

143

144

145
Figure 1: Sensor reference system and location. (A) Sensor and cluster with their reference system. (B) Example of all sensors and clusters mounted. The spinal segments (lumbar, low thoracic, upper thoracic and cervical) are defined by sensors pairs (S1L1, L1-T6, T6-T1, and T1-SK).

The protocol included five functional movements inspired by the BASMI protocol: trunk flexion, trunk extension, trunk lateral flexion, cervical rotation and cervical flexion/extension (Fig. 2).

Peer) reviewing PDF | (2020:07:50633:2:0:NEW 20 Nov 2020) 
146 Due to technical contingencies, cervical flexion/extension was collected on five out of eleven

147 participants. Only lumbar, lower thoracic and upper thoracic motions (sensors S1, L1, T6, T1)

148 were recorded for trunk movements (Fig. 2A-C). Cervical motions were excluded from the

149 analysis as not part of the BASMI protocol, and due to the uncontrolled compensatory head

150 movements performed by the participants whilst performing trunk lateral bending. During the

151 cervical movements (Fig. 2D and E), only the cervical spine sensors (T1 and SK) were used for

152 the analysis (Fig. 1B).

153

154

155

156

157

158

159

160

161

162

163

164

165

166

167

168

169

170

171 The segment angles were defined as the orientation ${ }_{G}^{B} q$ of a spinal body segment $(B)$ with respect

172 to the global reference system $G$ (Fig. 3B). To do this, three transformations were applied (Eq.

173 1): i) the rotation of the fixed $(F)$ reference system with respect to the global $(G)$ reference 
174 system $\left({ }_{G}^{F} q\right)$, ii) the sensor $(S)$ rotation with respect to the fixed $(F)$ reference system $\left({ }_{F}^{S} q\right)$, and iii)

175 the spinal segment $(B)$ rotation with respect to the sensor $(S)$ reference system $\left({ }_{S}^{B} q\right)$.

$$
{ }_{G}^{B} q={ }_{G}^{F} q{ }_{F}^{S} q{ }_{S}^{B} q
$$

176

177

178

179

180

181

182

183

184

185

186

187

188

189

190

191

192

193

194

195

196

197

198

FIGURE 3 here

Figure 3: Kinematic constraint axes and reference systems. (A) Representation of sensor axes and kinematic constraints axes. The sensor local reference system is shown in green, the kinematic constraints axes are shown in red, and the global reference system planes are shown in black. (B) Segment angle $\left({ }_{G}^{S} q_{1}\right)$ and a joint angle $\left({ }_{S}^{S} q_{2}-1\right)$ orientation. The sensor angle orientation ( ${ }_{F}^{S} q_{1}$ ) with respect to the fixed reference system (blue), the fixed reference system (orange) orientation $\left({ }_{G}^{F} q_{1}\right)$ with respect to the global reference system, and body reference system orientation with respect to the sensor reference system $\left({ }_{S}^{B} q_{2}\right)$ are shown.

The transformation ${ }_{S}^{B} q$ is representative of the soft tissue artefact's contribution to the measure, which was not experimentally measured in this study. For this reason, the sensor $(S)$ and body $(B$ ) were hypothesised being a rigid body with a constant relative orientation $\left({ }_{S}^{B} q\right)$, and aligned reference systems (Fig. 3B). The term ${ }_{S}^{B} q$ is therefore a quaternion with no rotation (Eq. 2), and the final ${ }_{G}^{B} q$ calculation can be simplified as follows (Eq. 3):

$$
\begin{aligned}
& { }_{S}^{B} q=[1,0,0,0] \\
& { }_{G}^{B} q={ }_{G}^{S} q={ }_{G}^{F} q{ }_{F}^{S} q
\end{aligned}
$$

The sensor orientation with respect to its fixed reference system $\left({ }_{F}^{S} q\right)$, was calculated internally by the IMU software. The magnetometer provided the local north direction for the heading calculation (yaw), the accelerometer gravity vector contributed to the tilt estimation (roll and pitch) and the gyroscope angular velocity completed the data for the orientation calculation through sensor fusion. The sensor reference system was set up with the $\hat{Z}$ axis parallel and opposite to the gravity vector, and the $\hat{X}$ axis aligned with the local magnetic north (Fig. 3B).

\section{IMU Kinematic Constraints and alignments.}

In the proposed method, different kinematic constraints were applied to correct for the vertical axis angular drift and improve the estimation of trunk segment angles. The kinematic constraints were applied from the end of the eight second calibration phase $\left(t_{z e r o}\right)$ throughout the whole 
199 recording for the sagittal and frontal plane functional movements. More specifically, the ${ }_{G}^{F} q$

200 rotation constrained the movement to lie on the global sagittal or frontal plane according to the

201 nature of the movement (see Appendix). This constraint assumes that the sensors reflect the

202 actual tilt of the spine in the sagittal and frontal planes, and that the sensor heading is always

203 aligned with the global reference system $\hat{X}$ axis. For the movement in the transversal plane, an

204 offset calculation was performed at $t_{z e r o}$, and this constant offset was subtracted throughout the

205 whole recording (see Appendix). This alignment assumes that the sensors reflect the actual tilt of

206 the spine in the sagittal and frontal planes, and that the spine axial rotation is zero at $t_{z e r o}$.

207 An optimisation analysis was also conducted to further improve the estimation of the spinal

208 segment and joint angles during lateral flexion. For each sensor, four different auxiliary axes

209 (Fig. 3A), in addition to the $\hat{z}$ axis, were created on the sensor sagittal plane ( $\hat{x} \hat{z}$ plane). The

210 optimisation identified the axis that generated the constraint producing the smallest measurement

211 error, and the same axis was used for all participants (see Appendix).

\section{Motion capture segment angles calibration.}

213 A set of marker clusters were attached to the IMUs to compare the alignment between their local

214 reference system with the IMU sensors local reference system (Fig. 1A). A virtual rigid body

215 was created for each cluster in the Qualisys Track Manager (QTM), and its orientation was

216 exported in rotation matrix format to be subsequently converted into quaternions.

217 To compare the clusters and the IMUs segment angles, any misalignments of the participant's

218 sagittal plane with respect to the $\hat{X} \hat{Z}$ plane of the motion capture global reference system (Fig.

$2193 \mathrm{~A}$ ) was compensated. A vertical rotation was imposed at the instant $t_{\text {zero }}$ on the motion capture

220 orientation to align the sensor on the global sagittal $(\hat{X} \hat{Z})$ plane. Optical motion capture systems

221 cannot drift and therefore kinematic constraints were not necessary. The same process was used

222 for all the functional movements, including the calculation of the spine segment angle.

223 Motion capture and IMU joint angles.

224 The joint angles of the spinal segments were obtained through a quaternion multiplication (Eq.

225 4).

${ }_{S}^{S} q_{2-1}={ }_{G}^{S} \bar{q}_{1}{ }_{G}^{S} q_{2}$ 
226 Where ${ }_{G}^{S} \bar{q}_{1}$ indicates the conjugate of the unit quaternion expressing the rotation of sensor 1 with

227 respect to the global reference system, ${ }_{G}^{S} q_{2}$ indicates the unit quaternion expressing the rotation

228 of sensor 2 with respect to the global reference system, and ${ }_{S}^{S} q_{2-1}$ is the quaternion expressing

229 the relative orientation of sensor 2 with respect to sensor 1 (Fig. 3B).

230 Flexion/extension, lateral flexion and axial rotation for segment and joint angles.

231 Finally, by using the convention from Crawford et al. (1999), the segment and joint angles were 232 expressed in quaternions and transformed into clinical angles.

$$
\begin{gathered}
\theta=\tan ^{-1}\left(\frac{\hat{x}_{y}}{\hat{x}_{x}}\right) \quad \varphi=\tan ^{-1}\left(\frac{\hat{x}_{y} \sin \theta+\hat{x}_{x} \cos \theta}{\hat{x}_{z}}\right) \\
F=\varphi \cos \theta \\
T=-\tan ^{-1}\left(\frac{-\hat{z}_{z} \sin \theta-\hat{y}_{z} \cos \theta}{\hat{z}_{z} \cos \theta-\hat{y}_{z} \sin \theta}\right)
\end{gathered}
$$

Where $\mathrm{F}$ is flexion/extension angle (Eq. 6, left), $\mathrm{L}$ is lateral flexion angle (Eq. 6, right), and $\mathrm{T}$ is axial rotation angle (Eq. 7). An example of such angle traces is shown in Figure 4A. It should be noted that $\hat{z}_{Z}$ has an opposite sign with respect to the original formulation because that axis in the reference system of this study is directed backwords.

FIGURE 4 here

Figure 4: Clinical angles and angle segmentation. (A) Clinical angles: flexion/extension angle in red, lateral flexion angle in green, axial rotation angle in blue. Example of joint angle segmentation for lumbar segment during flexion (B), lower thoracic segment during lateral flexion (C), cervical segment during cervical flexion/extension (D).

\section{Range of motion and continuous spinal kinematics data segmentation.}

Using a custom Matlab function (MATLAB R2017b, MathWorks inc., Natick, MA, USA), segment and joint angles were processed, and spinal ROM and kinematics were calculated. The ROM data segmentation consisted of finding the angles between the anatomical reference position and the maximum ROM, for both sensors (segment angles), and spinal segments (joint angles). The only exception was cervical flexion/extension where ROM was calculated as the angle between maximum cervical flexion and maximum cervical extension. Since each 
248 movement was repeated three times and each repetition consisted of two phases (performing the

249 movement and then returning to the reference position), flexion and extension produced 6

250 segments (Fig. 4B), lateral flexion and cervical rotation produced 12 segments (6 left and 6 right,

251 Fig. 4C), and cervical flexion/extension only 3 segments (Fig. 4D). The segmentation points

252 were calculated on IMU data, and the same points were adopted for motion capture data

253 segmentation. Joint and segment angle ROM for both IMU and motion capture were obtained by

254 subtracting the angular value at the beginning from the angular value at the end of every

255 segment. This produced a ROM value for every segment. Finally, the absolute value was

256 calculated for each of these ROMs. For the kinematic data analysis, the angular traces were

257 segmented between the onset of the first movement and the end of the last movement (see the red

258 lines in Fig. 4B, C, and D) to evaluate the sensors performance across a complete functional

259 movement.

\section{Sample size calculation}

261 The Bland-Altman sample size calculation was performed on data collected on six participants.

262 The statistical power analysis for Bland-Altman (MedCalc 2020) was set considering a type I

263 error (alpha) of 0.05 and a type II error (beta) of 0.20 . The maximum difference between IMU

264 and motion capture measurement was set to $2^{\circ}$ for the sagittal plane, $8^{\circ}$ for the frontal plane and

$2654^{\circ}$ for the transverse plane, considering previous studies carried out with similar technology on

266 axSpA patients (Aranda-Valera et al. 2020). The sample size calculation yielded three different

267 sample sizes for the three anatomical planes: 195 samples for the sagittal plane, 114 sample for

268 the frontal plane and 73 sample for the transverse plane. Each range of motion measurement was

269 considered as an individual sample due to the intra- and inter-variability of the measure. For the

270 same reason a Bland-Altman repeated measure correction was not applied.

271 Statistical analysis.

272 The validity of spine ROM values was assessed using correlation and Bland-Altman analyses.

273 These tests were performed to examine the correlation, median bias and limits of agreement

274 (95\% confidence interval, 1,45 IQR (interquartile range)) between the ROM calculated via the

275 IMU and motion capture system. 
276 The relative reliability of the spine ROM values was assessed using the intraclass correlation

277 coefficient $\mathrm{ICC}_{2,1}$ (Two-way random effects, absolute agreement, and single rater). ICC values

278 between 0.6 and 0.8 represented a good level of agreement, and $>0.8$ represented excellent

279 agreement.

280 The Kolmogorov-Smirnov normality test was conducted to assess the distribution of the segment

281 and joint range of motion values. After the normality test, it was chosen to perform a

282 nonparametric analysis as the data were not normally distributed.

283 The absolute reliability of the spine ROM values was assessed using standardised error

284 measurement (SEM), calculated as

285

$$
S E M=s \cdot d_{\text {pooled }} \cdot \sqrt{1-I C C}
$$

286

287

288

289

290

291

292

293

294

295

and minimum detectable change (MDC), calculated as

$$
M D C=S E M \cdot 1.96 \cdot \sqrt{2}
$$

ROM mean absolute percentage error (MAPE) was also presented in the results. For the continuous spinal kinematic analysis, the root mean square error (RMSE), maximum absolute error (MAE), and Spearman correlation coefficient $(\rho)$ were calculated for both segment and joint angles. Finally, the distribution of absolute and percent error frequency for segment and joint angles were calculated and plotted for visual analysis.

\section{Results}

\section{Analysis of spinal range of motion.}

The spinal ROM mean and SD for all participants are reported in Table 2 and Table 3 for all the trials and all the sensors. Every table contains data of both motion capture and IMU.

Table here 2

Table here 3

The overall mean ROM measurement of segment and joint angles across all movements had a small and comparable bias $\left(\leq 0.5^{\circ}\right)$, whilst the overall mean limits of agreement proved to be on average $20 \%$ higher for the joint compared to segment angle ROM (Table 4 and Table 5). The 
302 lumbar and thoracic ROM measured in the sagittal plane had the smallest bias $\left(\leq 0.6^{\circ}\right)$ and limits

303 of agreement $\left(\leq 1.9^{\circ}\right)$, whilst the measurements during lateral flexion showed smaller bias $(\leq$ $\left.3041.0^{\circ}\right)$ and much larger limits of agreement $\left(\leq 6.4^{\circ}\right)$ (Table 5). The cervical rotation joint angle

305 (transversal plane) had bias close to zero $0.0^{\circ}$ but the limits of agreement were much larger $(\leq$

$3062.3^{\circ}$ ), resulting in greater variability of the data (Table 5).

307

308

309

310

311

312

313

314

315

316

317

318

319

320

321

322

323

324

325

326

327

328

329

330

331

332

333

334

Table 4 here

Table 5 here

The calculation of spinal ROM in the sagittal plane showed the lowest measurement error, followed by the cervical rotation and lateral flexion ROMs (Table 4 and Table 5). The highest overall measurement error was observed in the frontal plane (lateral flexion) for both segment $\left(0.5 \pm 2.4^{\circ}\right)$ and joint $\left(0.2 \pm 4.8^{\circ}\right)$ angles, and in cervical flexion/extension segment angles $(0.5 \pm$ $2.5^{\circ}$ ) (Table 4 and Table 5). This trend was confirmed in the Bland-Altman analysis, where $100 \%$ of the segment flexion (0.8\% MAPE), 97\% of segment extension 2.6\% MAPE), and 96\% of segment lateral flexion (3.5\% MAPE) fell within the $10 \%$ threshold. Comparable data distribution (within 10\% threshold) and MAPE values were found for the joint angles, but with higher percent errors, especially for the lateral flexion, which led to fewer data $(40 \%)$ lying within the $10 \%$ percent error threshold (Fig. 5C). In fact, during the lateral flexion, the limits of agreement were relatively high across all spinal joint angles, reaching a maximum of $6.4^{\circ}$ for the lumbar segment (Table 5), whereas the lateral flexion segment angles showed a maximum limit of agreement of $2.3^{\circ}$ (Table 4 ).

FIGURE 5 here

Figure 5: Bland-Altman analysis. Comparison of IMU to motion capture joint angles ROM for flexion (A), extension (B), lateral flexion (C), cervical rotation (D), cervical flexion/extension (E). Cervical rotation segment angles (F) were also added. A$\mathrm{C}$ represent the lumbar segment (cyan), lower thoracic segment (black), and upper thoracic segment (green). D and E represent the cervical segment (blue). F represents the head sensor SK (blue). All the plots show: median bias (black line), limits of agreement (1.45 IQR, black dotted lines), and 10\% error threshold (red dotted lines).

The relative reliability analysis $\left(\mathrm{ICC}_{2,1}\right)$ showed excellent agreement for both segmental (Table 4) and joint angles (Table 5). The absolute reliability analysis resulted in MDC values ranging between $1.3^{\circ}$ and $2.9^{\circ}$ for the segmental angles, and $2.1^{\circ}$ and $5.7^{\circ}$ for joint angles. In both cases, spinal flexion and extension movement showed the lowest MDC values whilst lateral flexion had the highest MDC values. 
335 Finally, the correlation analysis on the ROM data showed that the IMUs measurements were

336 highly and significantly correlated with motion capture measurements (on average $r>0.97$ for

337 both segment and joint angles), with the lowest being the lateral flexion joint angle $(\mathrm{r}=0.95, \mathrm{p}<$

$3380.001)$.

339 A Kolmogorov-Smirnov normality test was conducted on both segment and joint angles for all

340 sagittal, frontal and transversal planes (Table 6). The transversal plane did not pass the normality 341 test.

\section{Kinematics analysis.}

344 Segment angle absolute error was less than $4^{\circ}$ for more than $90 \%$ of the traces across all the movements, with the exception of cervical rotation, for which was about $85 \%$. More specifically, the sagittal plane angle measures were the most accurate, showing an absolute error smaller than

$3472^{\circ}$ and percent error smaller than $10 \%$ for more than $66 \%$ of the data (Fig. 6A and B). Similarly, 348 all measurements of joint angle traces, excluding cervical rotation, had an absolute error lower 349 than $4^{\circ}$ for $87 \%$ of the data (Fig. 6C). The lateral flexion analysis presented two different 350 pictures for absolute errors and percent errors. Absolute error frequency showed a constant decrease in classes containing greater errors for both segments and joint angles (Fig. 6A and C), whereas the percent error frequency was more evenly distributed, with lateral flexion and cervical rotation even showing an increase for the class err $>40 \%$ (Fig. 6B and D). On average, with cervical rotation excluded, the correlation coefficient between IMUs and motion capture segment angle traces (Table 4) was very high $(\rho=0.99)$. The RMSE was low for all the sensors, for all the movements $\left(\sim 1.5^{\circ}\right)$, with $2.5^{\circ}$ being the highest value recorded for segment T6 during flexion and extension (Table 4). There was high variability between sensors and movements, with an MAE ranging from $1.1^{\circ}$ to $5.4^{\circ}$, also MAE values were higher for movements with greater ROM (Table 4).

The joint angles traces (Table 5) measured via IMUs had very high correlation coefficients compared with the motion capture analogous, the only exception being upper thoracic for lateral flexion $(\rho=0.48)$. The RMSE was consistently low across all spinal segments with the highest value recorded on upper thoracic during lateral flexion $\left(2.9 \pm 1.3^{\circ}\right)$. Similarly, the MAE for all 
364 joint angles traces was low, except for the cervical flexion/extension angle $\left(6.1 \pm 1.0^{\circ}\right)$ and the

365 lateral flexion angle which was consistently high across all spinal segments $\left(5.2 \pm 2.0^{\circ}\right)$.

366

367

368

369

370

371

372

373

374

375

376

377

378

379

380

381

382

383

384

385

386

387

388

389

390

391

392

393
FIGURE 6 here

Figure 6: Percentage of frames error analysis. Percentages of frames associated with a specific error. Errors are divided into 4 error classes $\left(\mathrm{err}<2^{\circ}, 2^{\circ}<\operatorname{err}<4^{\circ}, 4^{\circ}<\operatorname{err}<6^{\circ}\right.$, err $>6^{\circ}$ ) for absolute errors (A and C), and 4 error classes $(\operatorname{err}<10 \%, 10 \%<$ err $<20 \%, 20 \%<\operatorname{err}<40 \%$, err $>40 \%$ ) for percent errors (B and D). All functional movements are represented. Segment results are shown in $\mathrm{A}$ and $\mathrm{B}$ while joint angle results are shown in $\mathrm{C}$ and $\mathrm{D}$.

\section{Discussion}

The purpose of this validation study was to determine whether IMUs are suitable to provide an accurate measure of the spinal ROM and joint kinematics for clinical assessments. A novel experimental protocol was proposed and evaluated during spinal functional movements informed by a metrology index (BASMI) widely adopted in rheumatology. The protocol allows for the measurement of both segment and joint angles of the spine, with the latter being the most relevant for clinical assessment. In the sagittal plane, joint angles were measured with high accuracy (mean bias and limits of agreement (1.96 SD) $0.4 \pm 1.6^{\circ}$ ), whilst the frontal plane proved to be more challenging due to experimental limitations, but overall yielded acceptable results (mean bias and limits of agreement (1.96 SD) $0.2 \pm 4.8^{\circ}$ ). The Bland-Altman analysis showed that the mean ROM bias for all segment and joint angles (mean absolute value $0.3^{\circ}$ for both) was strong, and the reliability analysis showed excellent agreement across the board. The IMU protocol provided an excellent measure of segment angle traces across the whole functional movements trials in terms of measurement error. RMSE was small for both sagittal plane $(1.7 \pm$ $\left.0.8^{\circ}\right)$ and frontal plane $\left(1.2 \pm 0.7^{\circ}\right)$.

While the authors believe the results in this study demonstrated low errors compared to the gold standard, five factors that influenced measurement error were identified: (1) segment or joint angle type, (2) plane of movement, (3) sensor location on the spine, (4) type of constraint adopted and (5) soft tissue artefacts.

Measurement error of segment angles was lower compared to joint angles due to joint angles being calculated as the angular difference between two neighbouring sensors, and therefore the measurement error of each sensor was combined. The kinematic constraints adopted proved to be 
394 excellent for the movements in the sagittal plane, but less effective for the movements in the 395 frontal plane. To address such challenges, future work should focus on a more sophisticated 396 constraint that accounts for the complex spinal movements (e.g. coupled motions), or

397 alternatively, a tailored Kalman filter could mitigate magnetic distortions and gyroscope drift.

398 The movements performed in the sagittal plane had a higher accuracy than the movements in the 399 frontal and transverse planes (Table 4 and Table 5, Fig. 6). This is due to the stabilising role of 400 the accelerometers on horizontal tilt, and the assumption that such movements are performed 401 with a negligible axial rotation of the spine. This facilitated the constraint of the medial-lateral 402 axis onto the frontal plane, which provided an excellent solution against vertical axis drift 403 rotations provoked by magnetic distortions.

404 Segment and joint cervical rotation angles had higher measurement errors than all other spinal 405 angles. This was due to methodological and experimental challenges which exposed cervical 406 rotation measurements to a greater drift. Firstly, axial rotation measurements on the vertical axis 407 rely on gyroscopes and magnetometers, and therefore no accelerometer stabilisation could be 408 used to improve the measurement. Also, kinematic constraints were unsuitable for this 409 movement, and sensor alignment was only possible in the sagittal plane during calibration. 410 Finally, the cervical rotation joint angle measurements (Fig. 5D) suffered from soft tissue 411 artefact on sensor T1, where the skin stretch produced rotations not representative of the cervical 412 spine movement. For this reason, it is advised to use the head segment angle (Fig. 5F) to measure 413 the cervical rotation, assuming that the trunk is still. Also, during cervical rotation, it is crucial to 414 monitor potential trunk compensatory movements especially at extreme cervical range of 415 motions. Other transverse plane movements, such as the thoracic axial rotation, were excluded 416 from the analysis as they were not part of the BASMI, and their measurement was affected by 417 high drift on the vertical axis, which could be corrected using the constrained method proposed.

418 Lastly, the higher limits of agreement registered for lateral flexion can be also attributed to two 419 independent factors: soft tissue artefact (skin stretch and muscle contraction generate rotations 420 out of the frontal plane) and axial rotation components contextual to the lateral flexion. These 421 two phenomena indicate that while the constraint adopted prevents angular drift around the 422 vertical axis, it is too simplistic to account for spine axial rotations. 
423 The results of this study compare well or outperform those from previous literature. Aranda-

424 Valera et al. (2018) developed an IMU-based (ViMove, dorsaVi, Melbourne, Australia) posture

425 assessment that showed similar strengths and weaknesses to the current protocol, but with errors

426 (RMSE) being 2.5 times higher for lumbar lateral flexion $\left(8.3^{\circ}\right)$, and almost 4 times higher for

427 cervical rotation $\left(9.4^{\circ}\right)$ with respect to our study. Additionally, Aranda-Valera et al. (2018) study

428 only examined lumbar and cervical segments while the current protocol assesses the entire spine.

429 However, Aranda-Valera et al. (2018) presented errors with respect to full-arc movements rather

430 than half-arc, which is the case for the lateral bending errors in this paper. Despite such

431 difference, the present paper collected normal data instead of involving patients, and analogous

432 movements' amplitudes might be much greater. A more recent study by Aranda-Valera et al.

433 (2020) proposed a concurrent criterion validity between IMUs (ViMove) and optical motion

434 capture system (UCOTrack) on a cohort of 70 axSpA patients. The ROM bias and limits of

435 agreement values were significantly higher than those found in this study. The lumbar

436 flexion/extension showed a bias five times higher $\left(5.5^{\circ}\right)$ and a LOA fifteen times higher $( \pm$

$43725.2^{\circ}$ ) than the lumbar flexion and extension bias and LOA shown in this study (Table 5).

438 Similarly, the cervical rotation measure had a higher bias $\left(1.2^{\circ}\right.$, more than twice higher $)$ and

439 LOA $\left(32.6^{\circ}\right.$, fourteen times higher). It needs to be noted that Aranda-Valera et al. (2020)

440 conducted a parametric analysis, whereas in this paper a nonparametric analysis is performed.

441 Also, Aranda-Valera et al. (2020) used the spinal assessment measures from Garrido-Castro et al.

442 (2012) as a gold standard, which have some differences when compared to the ViMove protocol,

443 and soft tissue artefacts might have had an impact on the measure. From a joint kinematics

444 perspective, Mjøsund et al. (2017) showed results that are comparable or better than this study,

445 but the authors calculated the segment angles as "relative to the line of gravity". The two studies

446 also have different approaches for what concerns vertical axis rotations, where Mjøsund et al.

447 (2017) calculate the angles as inclinometer measurements on single planes, which mask rotations

448 around the vertical axis, this study adopts kinematic constraints to prevent rotations around the

449 vertical axis. Despite this difference in the methodology, the results of this study are comparable

450 to Mjøsund et al. (2017), showing a slightly higher RMSE $\left(0.9^{\circ}\right)$ and standard deviation $\left(0.5^{\circ}\right)$.

451 This is likely due the fact that the gold standard measure in Mjøsund et al. (2017) was affected

452 by the same bias, and therefore the movement on other planes are combined within the measure.

453 The results from this study compare well with literature that adopted tailored Kalman filter or 
454 kinematic constraints. The largest error in this study (cervical flexion/extension) had a RMSE

$4550.7^{\circ}$ smaller than that produced by Seel et al. (2014), and $0.8^{\circ}$ smaller than Cooper et al. (2009), 456 demonstrating an improvement of $\sim 20 \%$. It should be noted that the slow spine movements in

457 this study will rely more on the stabilization of the accelerometers, whereas the high pace but

458 less broad movements of walking and running rely more on the quick response of the

459 gyroscopes. A recent study by Lee \& Jeon (2019) obtained very promising results by excluding

460 the magnetometer data and applying kinematic constraints, reaching a RMSE of $1.58^{\circ}$. These

461 results are not fully comparable with the present study, as the Kalman filter used in the presented

462 was not customisable and raw data were inaccessible to the authors.

463 While this IMU protocol could be used for most patients, severe AS cases suffer from restricted 464 ROM and hence the measurement error found in this study could be close to the limited spinal 465 ROM of these patients. Therefore, future clinical applications of IMU systems must consider the 466 restricted movement of the targeted population and evaluate if the system is still viable by 467 running a clinical validation on patients. While data collection using the method presented here

468

469

470

471

472

473

474

475

476

477

478

479

480

481

482 may take 10 minutes longer to perform than the BASMI, this protocol has several advantages such as reduced human error and more detailed measures within and between spinal segments. This is highly valuable and accurate information to be used in clinical settings, as it would enable an immediate and automated comparison against historical and benchmark values. In fact, the BASMI loses focus on the spine compared to the proposed sensor protocol which accurately measures segment-specific biomechanical changes that could highlight potential intervertebral fusion levels. Additionally, cervical rotation and intermalleolar distance in the BASMI are measured while lying in a supine position, which can be painful for patients with severe AS and does not mimic cervical rotation as it would normally be performed (during standing). On the other hand, the proposed protocol lacks a full clinical validation and reliability analysis, and there are measures performed by the BASMI that are not measurable with this IMU protocol, such as postural data (tragus to wall) and ROM data form hips (intermalleolar distance).

\section{Conclusions}

In summary, this new IMU-based protocol is capable of accurately measuring spinal ROM and kinematics. This study also demonstrates that IMU technologies are a promising alternative to 
483

484

485

486

487

488

489

490

491

492

493

494

495

496

497

498

499

500

501

502

503

504

505

506

507

508

509

510

511

512

513

514

515

516

517

518

519

assess the axial status and its changes. The kinematic constraints adopted seem to have a key role in containing the measurement error, when compared to state of the art motion capture. This validation sets the ground to construct a mobility index for axSpA based on automated measures with better metric properties. Furthermore, the protocol's simplicity has potential to make its implementation in clinical setting possible and a potentially viable solution to integrate or substitute current clinical methods in the future. However, a clinical validation of the presented protocol on axSpA patients is needed to ensure the clinical usability of such means.

\section{Acknowledgments}

I want to thank Dr Steffi Colyer, Dr Valentina Camomilla, James Cowburn and Nicos

Haralabidis for their valuable suggestions on the paper.

\section{References}

Ahmadi A, Rowlands DD, and James DA. 2010. Development of inertial and novel marker-based techniques and analysis for upper arm rotational velocity measurements in tennis. Sports Eng:12:179-188.

Akkoc N. 2018. Personalized Axial Spondyloarthritis Care. Current Treatment Options in Rheumatology. 10.1007/s40674-018-0094-2

Akkoc N, Direskeneli H, Erdem H, Gul A, Kabasakal Y, Kiraz S, Balkan Tezer D, Hacibedel B, and Hamuryudan V. 2015. Direct and indirect costs associated with ankylosing spondylitis and related disease activity scores in Turkey. Rheumatol Int 35:1473-1478. 10.1007/s00296-015-3236-y

Aranda-Valera IC, Cuesta-Vargas A, Garrido-Castro JL, Gardiner P, López-Medina C, Machado PM, Condell J, Connolly J, Williams J, Muñoz Esquivel K, O'Dwyer T, Castro-Villegas M, González-Navas C, and Collantes-Estevez E. 2020. Measuring Spinal Mobility Using an Inertial Measurement Unit System: A Validation Study in Axial Spondyloarthritis. Diagnostics 10:426. 10.3390/diagnostics10060426

Aranda-Valera IC, Garrido-Castro JL, Sánchez I, J G, Gardiner P, Machado PM, and Collantes-Estevez E. 2018. FRI0202 Inertial motion sensors using the vimove (C) system is a valid method to assess spinal mobility in patients with axial spondyloarthritis. In: Dis AR, editor: Annals of the Rheumatic Diseases. p 642.642-643.

Baraliakos X, and Braun J. 2015. Non-radiographic axial spondyloarthritis and ankylosing spondylitis: what are the similarities and differences? RMD Open 1:e000053. 10.1136/rmdopen-2015-000053

Beange KHE, Chan ADC, Beaudette SM, and Graham RB. 2019. Concurrent validity of a wearable IMU for objective assessments of functional movement quality and control of the lumbar spine. J Biomech 97:109356. 10.1016/j.jbiomech.2019.109356

Calin A, Garrett S, Whitelock H, Kennedy LG, O'Hea J, Mallorie P, and Jenkinson T. 1994. A new approach to defining functional ability in ankylosing spondylitis: the development of the Bath Ankylosing Spondylitis Functional Index. The journal of rheumatology 21:2281-2285.

Calvo-Gutierrez J, Garrido-Castro JL, Gonzalez-Navas C, Castro-Villegas MC, Ortega-Castro R, LopezMedina C, Font-Ugalde P, Escudero-Contreras A, and Collantes-Estevez E. 2016. Inter-rater 
reliability of clinical mobility measures in ankylosing spondylitis. BMC Musculoskelet Disord 17:382. 10.1186/s12891-016-1242-1

Cappello A, Stagni R, Fantozzi S, and Leardini A. 2005. Soft tissue artifact compensation in knee kinematics by double anatomical landmark calibration: performance of a novel method during selected motor tasks. IEEE Trans Biomed Eng 52:992-998. 10.1109/TBME.2005.846728

Charry E, Umer M, and Taylor S. 2011. Design and Validation of an Ambulatory Inertial System for 3-D Measurements of Low Back Movements. Intelligent Sensors, Sensor Networks and Information Processing (ISSNIP).

Chhikara A, McGregor AH, Hadjilucas L, Bello F, and Rice AS. 2010. Quantitative Assessment of the Motion of the Lumbar Spine and Pelvis with Wearable Inertial Sensors. 2010 International Conference on Body Sensor Networks. p 9-15.

Cooksey R, Husain MJ, Brophy S, Davies H, Rahman MA, Atkinson MD, Phillips CJ, and Siebert S. 2015. The Cost of Ankylosing Spondylitis in the UK Using Linked Routine and Patient-Reported Survey Data. PLOS ONE 10:e0126105. 10.1371/journal.pone.0126105

Cooper G, Sheret I, McMillan L, Siliverdis K, Sha N, Hodgins D, Kenney L, and Howard D. 2009. Inertial sensor-based knee flexion/extension angle estimation. J Biomech 42:2678-2685. 10.1016/j.jbiomech.2009.08.004

Cox MW, McCarthy M, Lemmon G, and Wenker J. 2001. Cervical Spine Instability: Clearance Using Dynamic Fluoroscopy. Current Surgery 58:96-100.

Crawford NR, Yamaguchi GT, and Dickman CA. 1999. A new technique for determining 3-D joint angles: the tilt/twist method. Clin Biomech 14:153-165.

Dagfinrud H, Kjeken I, Mowinckel P, Hagen KB, and Kvien TK. 2005. Impact of functional impairment in ankylosing spondylitis: impairment, activity limitation, and participation restrictions. J Rheumatol:32;516-523.

de Vries WH, Veeger HE, Baten CT, and van der Helm FC. 2009. Magnetic distortion in motion labs, implications for validating inertial magnetic sensors. Gait Posture 29:535-541. 10.1016/j.gaitpost.2008.12.004

Ernst MJ, Rast FM, Bauer CM, Marcar VL, and Kool J. 2013. Determination of thoracic and lumbar spinal processes by their percentage position between C7 and the PSIS level. BMC Res Notes 6:58. 10.1186/1756-0500-6-58

Favre J, Jolles BM, Aissaoui R, and Aminian K. 2008. Ambulatory measurement of 3D knee joint angle. Journal of Biomechanics 41:1029-1035. 10.1016/j.jbiomech.2007.12.003

Garrido-Castro J, Concha-Aranda I, Gardiner P, Machado P, Williams J, and Collantes-Estevez E. 2018. AB0867 Axial spondyloarthritis posture assessment using inertial sensors. Annals of the Rheumatic Diseases 77:1561. 10.1136/annrheumdis-2018-eular.3903

Garrido-Castro JL, Medina-Carnicer R, Schiottis R, Galisteo AM, Collantes-Estevez E, and Gonzalez-Navas C. 2012. Assessment of spinal mobility in ankylosing spondylitis using a video-based motion capture system. Manual Therapy 17:422-426. 10.1016/j.math.2012.03.011

Haglund E, Bremander A, Bergman S, Jacobsson LT, and Petersson IF. 2013. Work productivity in a population-based cohort of patients with spondyloarthritis. Rheumatology (Oxford) 52:17081714. 10.1093/rheumatology/ket217

Jenkinson TR, Mallorie PA, Whitelock HC, Kennedy LG, Garrett SL, and Calin A. 1994. Defining spinal mobility in ankylosing spondylitis (AS). The Bath AS Metrology Index. The journal of rheumatology 21:1694-1698.

Kawalec P, and Malinowski K. 2015. Disease activity, quality of life and indirect costs of reduced productivity at work, generated by Polish patients with ankylosing spondylitis. Reumatologia 53:301-308. 10.5114/reum.2015.57634 
Kruger K, von Hinuber U, Meier F, Tian H, Bohm K, Jugl SM, Borchert K, Meise D, Konig C, and Braun S. 2018. Ankylosing spondylitis causes high burden to patients and the healthcare system: results from a German claims database analysis. Rheumatol Int 38:2121-2131. 10.1007/s00296-0184124-z

Kulig K, Landel RF, and Powers CM. 2004. Assessment of Lumbar Spine Kinematics Using Dynamic MRI: A Proposed Mechanism of Sagittal Plane Motion Induced by Manual Posterior-to-Anterior Mobilization. J Orthop Sports Phys Ther 34:57-64.

Laidig D, Müller P, and Seel T. 2017a. Automatic anatomical calibration for IMU-based elbow angle measurement in disturbed magnetic fields. Current Directions in Biomedical Engineering 3. 10.1515/cdbme-2017-0035

Laidig D, Schauer T, and Seel T. 2017b. Exploiting Kinematic Constraints to Compensate Magnetic Disturbances when Calculating Joint Angles of Approximate Hinge Joints from Orientation Estimates of Inertial Sensors. International Conference on Rehabilitation Robotics. London. p 971976.

Lee JK, Desmoulin GT, Khan AH, and Park EJ. 2011a. Comparison of 3D spinal motions during stair-climbing between individuals with and without low back pain. Gait Posture 34:222-226. 10.1016/j.gaitpost.2011.05.002

Lee JK, Desmoulin GT, Khan AH, and Park EJ. 2011b. A Portable Inertial Sensing-based Spinal Motion Measurement System for Low Back Pain Assessment. Annual International Conference of the IEEE EMBS. Boston: IEEE. p 4737-4740.

Lee JK, and Jeon TH. 2019. Magnetic Condition-Independent 3D Joint Angle Estimation Using Inertial Sensors and Kinematic Constraints. Sensors (Basel) 19. 10.3390/s19245522

Martindale JH, Sutton CJ, and Goodacre L. 2012. An exploration of the inter- and intra-rater reliability of the Bath Ankylosing Spondylitis Metrology Index. Clin Rheumatol 31:1627-1631. 10.1007/s10067012-2057-6

Martinus H. 2017. JustSketchMe, JustSketchMe Posing Tool. Available at https://justsketch.me/ (accessed 11 September 2019.

McGonagle D, and Benjamin M. 2009. Entheses, enthesitis and enthesopathy. Arthritis Research Campaign (ARC) 4.

Mjøsund HL, Boyle E, Kjaer P, Mieritz RM, Skallgard T, and Kent P. 2017. Clinically acceptable agreement between the ViMove wireless motion sensor system and the Vicon motion capture system when measuring lumbar region inclination motion in the sagittal and coronal planes. $B M C$ Musculoskelet Disord 18:124. 10.1186/s12891-017-1489-1

Mousavi SJ, Tromp R, Swann MC, White AP, and Anderson DE. 2018. Between-session reliability of optoelectronic motion capture in measuring sagittal posture and 3-D ranges of motion of the thoracolumbar spine. Journal of Biomechanics 8. 10.1016/j.jbiomech.2018.08.033

Nuesch C, Roos E, Pagenstert G, and Mundermann A. 2017. Measuring joint kinematics of treadmill walking and running: Comparison between an inertial sensor based system and a camera-based system. J Biomech 57:32-38. 10.1016/j.jbiomech.2017.03.015

Rafia R, Ara R, Packham J, Haywood KL, and Healey EL. 2012. Healthcare costs and productivity losses directly attributable to ankylosing spondylitis. Clin Exp Rheumatol:30: 246-253.

Raine C, and Keat A. 2018. Axial spondyloarthritis. Medicine 46:231-236. 10.1016/j.mpmed.2018.01.005

Rezvani A, Ergin O, Karacan I, and Oncu M. 2012. Validity and reliability of the metric measurements in the assessment of lumbar spine motion in patients with ankylosing spondylitis. Spine (Phila Pa 1976) 37:E1189-1196. 10.1097/BRS.0b013e31825ef954

Seel T, Raisch J, and Schauer T. 2014. IMU-based joint angle measurement for gait analysis. Sensors (Basel) 14:6891-6909. 10.3390/s140406891 
614 Slajpah S, Kamnik R, and Munih M. 2014. Kinematics based sensory fusion for wearable motion

615

616

617

618

619

620

621

622

623

624

625

626 assessment in human walking. Comput Methods Programs Biomed 116:131-144. 10.1016/j.cmpb.2013.11.012

Takeda R, Tadano S, Natorigawa A, Todoh M, and Yoshinari S. 2009. Gait posture estimation using wearable acceleration and gyro sensors. J Biomech 42:2486-2494. 10.1016/j.jbiomech.2009.07.016

Taurog JD, Chhabra A, and Colbert RA. 2016. Ankylosing Spondylitis and Axial Spondyloarthritis. N Engl J Med 374:2563-2574. 10.1056/NEJMra1406182

Walsh JA, Song X, Kim G, and Park Y. 2018. Healthcare Utilization and Direct Costs in Patients with Ankylosing Spondylitis Using a Large US Administrative Claims Database. Rheumatol Ther 5:463474. 10.1007/s40744-018-0124-4 
627 Appendix

628 This appendix contains the initial sensor alignment on the sagittal plane, and the constraints

629 applied for every specific movement.

630 Flexion-extension constraint (trunk flexion, trunk extension and cervical flexion/extension).

631 The $\hat{y}$ axis unit vector projection on the $\hat{X} \hat{Y}$ plane was found, and its orientation $\alpha$ with respect to 632 the $\hat{X}$ axis was calculated through the four-quadrant inverse tangent (Eq. 6). This was done for all 633 the orientations at all the time points $t$. Successively, the angles $\beta_{t}$ between the projection and 634 the $\hat{Y}$ axis were calculated by subtracting $\pi / 2$ from $\alpha_{t}$ (Eq. 7).

$$
\begin{gathered}
\alpha_{t}=\tan ^{-1}\left(\hat{y}_{y}(t) / \hat{y}_{x}(t)\right) \\
\beta_{t}=\alpha_{t}-\pi / 2
\end{gathered}
$$

635 Then, the quaternion angle by which the sensor orientation needs to be rotated around the fixed 636 axis $\hat{Z}$ was:

$$
{ }_{G}^{F} q=q_{\beta Z t}=\left[\cos \left(-\frac{\beta_{t}}{2}\right), 0,0, \sin \left(-\frac{\beta_{t}}{2}\right)\right]
$$

637 Finally, the reorientation was performed by multiplying every quaternion ${ }_{G}^{F} q$ by the 638 corresponding sensor orientation quaternion ${ }_{F}^{S} q$ (Eq. 9).

$$
{ }_{G}^{S} q={ }_{G}^{F} q{ }_{F}^{S} q
$$

\section{Lateral flexion constraints (trunk lateral flexion).}

640 The angle $\gamma$ between the local $\hat{z}$ axis vector and its projection on the $\hat{X} \hat{Y}$ plane was calculated at $641 t_{\text {zero }}$ (Eq. 10), then a unit vector $\hat{h}_{0}=\left[\begin{array}{lll}0 & 0 & 1\end{array}\right]$ was rotated around its $\hat{y}$ axis by an angle of $-\gamma$ (Eq. $64211)$.

$$
\begin{gathered}
\gamma=\tan ^{-1}\left(\hat{z}_{z}\left(t_{\text {zero }}\right) / \sqrt{\hat{z}_{x}\left(t_{\text {zero }}\right)^{2}+\hat{z}_{y}\left(t_{\text {zero }}\right)^{2}}\right) \\
\hat{h}_{0,-\gamma}=\left[\begin{array}{ccc}
\cos (-\gamma) & 0 & \sin (-\gamma) \\
0 & 1 & 0 \\
-\sin (-\gamma) & 0 & \cos (-\gamma)
\end{array}\right] \cdot \hat{h}_{0}
\end{gathered}
$$


643 The quaternion rotation ${ }_{F}^{S} q$, representing the sensor orientation in space with respect to its fixed 644 reference system, was then applied to $\hat{h}_{0,-\gamma}$. The resulting unit triad has at $t_{\text {zero }}=8 \mathrm{~s}$ its $\hat{z}$ axis 645 aligned with the $\hat{X} \hat{Y}$ plane, that is now called $\hat{h}_{t}$ (Eq. 12).

$$
\hat{h}(t)={ }_{F}^{S} q \hat{h}_{0,-\gamma F}^{S} \bar{q}
$$

646 The orientation $\alpha_{t}$ of the projection of vector $\hat{h}(t)$ on the $\hat{X} \hat{Y}$ plane was then calculated (Eq. 13). 647 This was done for all orientations at all time points $t$. Successively, the angles $\beta_{t}$ between the 648 projection and the negative $\hat{X}$ axis were calculated by subtracting $\pi$ to $\alpha_{t}$ (Eq. 14).

$$
\begin{gathered}
\alpha_{t}=\tan ^{-1}\left(\hat{h}_{y}(t) / \hat{h}_{x}(t)\right) \\
\beta_{t}=\alpha_{t}-\pi
\end{gathered}
$$

649 Then, the quaternion angle by which the sensor orientation needs to be rotated around the fixed 650 axis $\hat{Z}$ was (Eq. 15):

$$
{ }_{G}^{F} q=q_{\beta Z t}=\left[\cos \left(-\frac{\beta_{t}}{2}\right), 0,0, \sin \left(-\frac{\beta_{t}}{2}\right)\right]
$$

651 Finally, the reorientation was performed by multiplying every quaternion ${ }_{G}^{F} q$ by the 652 corresponding sensor orientation quaternion ${ }_{F}^{S} q$ (Eq. 16).

$$
{ }_{G}^{S} q={ }_{G}^{F} q \underset{F}{S} q
$$

653 The procedure above describes the case in which the horizontal axis ( $\hat{h}$ axis) is adopted as a 654 constraint. To adopt any of the other 4 axes $\left(\hat{z}_{2}, \hat{z}, \hat{z}_{-1}, \hat{z}_{-2}\right)$ as a constraint, Equation 11 and 12 655 become respectively: Equation 17 left and right (case $\hat{z}_{2}$ axis), Equation 18 left and right (case $\hat{z}$ 656 axis), Equation 19 left and right (case $\hat{z}_{-1}$ axis), Equation 20 left and right (case $\hat{z}_{-2}$ axis).

$$
\begin{array}{ccc}
\hat{h}_{0,+\gamma}=\left[\begin{array}{ccc}
\cos (+\gamma) & 0 & \sin (+\gamma) \\
0 & 1 & 0 \\
-\sin (+\gamma) & 0 & \cos (+\gamma)
\end{array}\right] \cdot \hat{h}_{0} & \hat{z}_{2}(t)={ }_{F}^{S} q \hat{h}_{0,+\gamma F} \bar{q} \\
\hat{h}_{0,0}=\left[\begin{array}{lll}
1 & 0 & 0 \\
0 & 1 & 0 \\
0 & 0 & 1
\end{array}\right] \cdot \hat{h}_{0} & \hat{z}(t)={ }_{F}^{S} q \hat{h}_{0,0}{ }_{F}^{S} \bar{q}
\end{array}
$$




$$
\begin{aligned}
& \hat{h}_{0,-2 \gamma}=\left[\begin{array}{ccc}
\cos (-2 \gamma) & 0 & \sin (-2 \gamma) \\
0 & 1 & 0 \\
-\sin (-2 \gamma) & 0 & \cos (-2 \gamma)
\end{array}\right] \cdot \hat{h}_{0} \quad \hat{z}_{-1}(t)={ }_{F}^{S} q \hat{h}_{0,-2 \gamma}{ }_{F}^{S} \bar{q} \\
& \hat{h}_{0,-3 \gamma}=\left[\begin{array}{ccc}
\cos (-3 \gamma) & 0 & \sin (-3 \gamma) \\
0 & 1 & 0 \\
-\sin (-3 \gamma) & 0 & \cos (-3 \gamma)
\end{array}\right] \cdot \hat{h}_{0} \quad \hat{z}_{-2}(t)={ }_{F}^{S} q \hat{h}_{0,-3 \gamma F} \bar{q}
\end{aligned}
$$

\section{Constraint optimisation (trunk lateral flexion):}

658 For the kinematic constraints application in the lateral flexion movement, the five axes $\hat{h}, \hat{z}_{2}, \hat{z}$, $659 \hat{Z}_{-1}, \hat{z}_{-2}$ (Equation 12, and Equations 17, 18, 19 and 20 right) were adopted as constraints (Fig.

$6603 \mathrm{~A}$ ), and an optimisation that minimised the ROM measurement error absolute value was

661 performed to find the best axes for the segment angles and the best axes pairs for the joint angles.

662 All five axes were tested for each segment angle, and considering that the joint angles represent

663 the relative angle between two neighbouring sensors, all twenty-five ( $5 * 5$ axes) combinations

664 were tested for the joint angles. Each of these axes was tested as a kinematic constraint, for every

665 sensor and every participant.

666 Mean and standard deviation were calculated for the ROM measurement error absolute values of 667 all sensors and participants, subsequently they were plotted against the five axes (segment 668 angles, Fig. A1A) and the twenty-five axes combinations (joint angles, Fig. A1B). The segment 669 angles constraints were numbered as follows: axis $\hat{z}_{-2}$ was constraint $1, \hat{z}_{-1}$ was constraint $2, \hat{h}$ 670 was constraint $3, \hat{z}$ was constraint $4, \hat{z}_{2}$ was constraint 5 . The 25 -constraint combination 671 numbering is shown in Table A1. The first 5 combined the lower sensor axis $\hat{Z}_{-2}$ with the upper

672 sensor axes going from $\hat{z}_{-2}$ to $\hat{z}_{2}$, the combinations from 6 to 10 combined the lower sensor axis $673 \hat{z}_{-1}$ with the upper sensor axes going from $\hat{z}_{-2}$ to $\hat{z}_{2}$, the same scheme repeats for all 25 674 constraints.

Figure A1A and B show mean and standard deviation distribution for ROM measurement error absolute values across respectively all axes (for segment angles) and all axes combinations (for 678 joint angles).

Figure A1: Constraint number optimisation. ROM measurement error absolute value, plotted against constraint number for all segment (A) and joint (B) angles. Mean (blue line) and standard deviation (orange circle) values are calculated across all participants, also the optimum value is shown (red diamond). 


\section{Cervical rotation alignment.}

687 The $\hat{Z}$ axis unit vector projection on the $\hat{X} \hat{Y}$ plane was found at $t_{\text {zero }}$, and its orientation $\alpha\left(t_{\text {zero }}\right)$ 688 with respect to the $\hat{X}$ axis was calculated through the four-quadrant inverse tangent (Eq. 21). 689 Successively, the angle $\beta\left(t_{\text {zero }}\right)$ between the projection and the negative $\hat{X}$ axis was calculated 690 by subtracting $\pi$ to $\alpha\left(t_{\text {zero }}\right)$ (Eq. 22$)$.

$$
\begin{gathered}
\alpha\left(t_{\text {zero }}\right)=\tan ^{-1}\left(\hat{z}_{y}\left(t_{\text {zero }}\right) / \hat{z}_{x}\left(t_{\text {zero }}\right)\right) \\
\beta\left(t_{\text {zero }}\right)=\alpha\left(t_{\text {zero }}\right)-\pi
\end{gathered}
$$

691 Then, the quaternion angle by which the sensor orientation needs to be rotated around the fixed 692 axis $\hat{Z}$ was (Eq. 23):

$$
{ }_{G}^{F} q\left(t_{\text {zero }}\right)=q_{\beta Z}\left(t_{\text {zero }}\right)=\left[\cos \left(-\frac{\beta\left(t_{\text {zero }}\right)}{2}\right), 0,0, \sin \left(-\frac{\beta\left(t_{\text {zero }}\right)}{2}\right)\right]
$$

693 Finally, the reorientation was performed by multiplying ${ }_{G}^{F} q\left(t_{\text {zero }}\right)$ by every sensor orientation 694 quaternion ${ }_{F}^{S} q$ (Eq. 24).

$$
{ }_{G}^{S} q={ }_{G}^{F} q\left(t_{\text {zero }}\right){ }_{F}^{S} q
$$

695

696

\section{Formulae statistical analysis.}

697 In the following formulae the subscript $A$ indicates the IMU data (Avanti), $Q$ the motion capture 698 system data (Qualisys), $r$ the movement repeat, $p$ the participant, $s$ the sensor, $n$ the total number 699 of movement repeats, $m$ the total number of participants, $o$ the total number of sensors, $t$ the 700 sample at a specific time, $w$ the total number of samples per recording.

Range of motion data

702 Measurement error for every participant (Eq. 25 left), and every sensor of every participant (Eq. 70325 right):

$$
\Delta_{A Q r, p}=R O M_{A r, p}-R O M_{Q r, p} \quad \Delta_{A Q s, r, p}=R O M_{A s, r, p}-R O M_{Q s, r, p}
$$

704 where $R O M$ indicates the angular range of motion. 
705 Nonparametric Bland-Altman analysis. Median bias and limits of agreement for all participants 706 (Eq. 26 and 28) and overall median bias and limits of agreement for all sensors of all participants 707 (Eq. 27 and 29):

$$
\begin{gathered}
B I A S_{r, p}=\frac{1}{2}\left(\Delta_{A Q r, p\lfloor(n+1) / 2\rfloor}+\Delta_{A Q r, p\lceil(n+1) / 2]}\right) \\
B I A S_{s, r, p}=\frac{1}{2}\left(\Delta_{A Q s, r, p\lfloor(n+1) / 2\rfloor}+\Delta_{A Q s, r, p\lceil(n+1) / 2\rceil}\right) \\
L O A_{r, p}=1.45 \cdot\left(Q_{3 \Delta_{A Q r, p}}-Q_{\left.1 \Delta_{A Q r, p}\right)}\right) \\
L O A_{s, r, p}=1.45 \cdot\left(Q_{3 \Delta_{A Q s, r, p}}-Q_{1 \Delta_{A Q s, r, p}}\right)
\end{gathered}
$$

708 where 1.45 computes the $95 \%$ nonparametric limits of agreement.

709 Mean absolute percentage error for all sensors of all participants (Eq. 30):

$$
M A P E_{s, r, p}=\frac{1}{o \cdot n \cdot m} \sum_{s=1}^{o} \sum_{r=1}^{n} \sum_{p=1}^{m}\left|\frac{\Delta_{A Q s, r, p}}{R O M_{Q s, r, p}}\right|
$$

\section{Kinematics data}

Measurement error for every angular sample of a recording (Eq. 31 left) and root mean square error for all the samples of a recording (Eq. 31 right):

$$
E_{A Q t}=y_{A t}-y_{Q t}
$$

$$
R M S E_{t}=\sqrt{\frac{\sum_{t=1}^{w}\left(E_{A Q t}\right)^{2}}{w}}
$$

713 where $y_{A t}$ and $y_{Q t}$ are the angle samples in time for respectively the IMU and motion capture 714 data.

715 Mean and standard deviation for all the root mean square errors for all the participants (Eq. 32 716 left and 33) and mean and standard deviation for all the root mean square errors for all the 717 sensors of all participants (Eq. 32 right and 34):

$$
\operatorname{mean}_{R M S E p}=\frac{\sum_{p=1}^{m} R M S E_{t}}{m} \quad \operatorname{mean}_{R M S E s, p}=\frac{\sum_{s=1}^{o} \sum_{p=1}^{m} R M S E_{t}}{o \cdot m}
$$




$$
\begin{gathered}
S D_{R M S E p}=\sqrt{\frac{\sum_{p=1}^{m}\left(R M S E_{t}-\operatorname{mean}_{R M S E}\right)^{2}}{m-1}} \\
S D_{R M S E s, p}=\sqrt{\frac{\sum_{s=1}^{o} \sum_{p=1}^{m}\left(R M S E_{t}-\text { mean }_{R M S E s, p}\right)^{2}}{o \cdot m-1}}
\end{gathered}
$$

718 Maximum absolute error for every angular sample of a recording (Eq. 35). Mean and standard 719 deviation for all the maximum absolute errors for all the participants (Eq. 36 left and 37) and 720 mean and standard deviation for all the maximum absolute errors for all the sensors of all 721 participants (Eq. 36 right and 38):

$$
\begin{gathered}
M A E_{A Q t}=\max \left(\left|E_{A Q t}\right|\right) \\
\operatorname{mean}_{M A E p}=\frac{\sum_{p=1}^{m} M A E_{A Q t}}{m} \quad \operatorname{mean}_{M A E s, p}=\frac{\sum_{s=1 p=1}^{o} \sum_{M A E_{A Q t} t}^{m}}{o \cdot m} \\
S D_{M A E p}=\sqrt{\frac{\sum_{p=1}^{m}\left(M A E_{A Q t}-\operatorname{mean}_{M A E p}\right)^{2}}{m-1}} \\
S D_{M A E s, p}=\sqrt{\frac{\sum_{s=1 p=1}^{o}\left(M A E_{A Q t}-m_{\text {mean }} \sum_{M A E s, p}\right)^{2}}{o \cdot m-1}}
\end{gathered}
$$

722 Spearman correlation coefficient for all the angular samples of a recording is $\rho_{A Q t}$.

723 Mean and standard deviation for all the Spearman correlation coefficients for all the participants 724 (Eq. 39 left and 40) and mean and standard deviation for all the Spearman correlation 725 coefficients for all the sensors of all participants (Eq. 39 right and 41):

$$
\operatorname{mean}_{\rho_{A Q t} p}=\frac{\sum_{p=1}^{m} \rho_{A Q t}}{m} \quad \operatorname{mean}_{\rho_{A Q t} s, p}=\frac{\sum_{s=1}^{o} \sum_{p=1}^{m} \rho_{A Q t}}{o \cdot m}
$$




$$
\begin{gathered}
S D_{\rho_{A Q t} p}=\sqrt{\frac{\sum_{p=1}^{m}\left(\rho_{A Q t}-\text { mean }_{\rho_{A Q t} p}\right)^{2}}{m-1}} \\
S D_{\rho_{A Q t} s, p}=\sqrt{\frac{\sum_{s=1}^{o} \sum_{p=1}^{m}\left(\rho_{A Q t}-\operatorname{mean}_{\rho_{A Q t} s, p}\right)^{2}}{o \cdot m-1}}
\end{gathered}
$$




\section{Table 1 (on next page)}

Verbal instructions given to the participant before performing each movement.

Verbal instructions given to the participant before performing each movement. 
2 Table 1: Verbal instructions given to the participant before performing each movement.

Flexion

Extension

Cervical

Flexion/Extension
Stand upright with your feet shoulder-width apart, arms down on the sides and keep your legs straight, then bend forward to touch your toes. When you feel you can't reach any further down, come back to the upright position. Perform this three times.

Stand upright with your feet shoulder-width apart and arms across your chest. Keeping your legs straight, lean backwards until you can't go any further while still maintaining your balance. Then come back to the upright position. Perform this three times.

Stand upright with your feet shoulder-width apart, arms down on the sides and keep your legs straight. Slide your left arm down along the side of your left leg until you cannot go any further, then come back upright. Don't bend forward or backwards, like are stuck between two walls. Perform this three times on the left side and three times on the right side.

Stand upright with your feet shoulder-width apart, arms down on the sides. Look left until you've reached the maximum range of motion, then return to the starting position. Remember to keep the shoulders and the rest of the body still (facing forward). Perform this three times on the left side and three times on the right side.

Stand upright with your feet shoulder-width apart, arms down on the sides. Tilt only your head forward until you can't go any further, then tilt your head all the way back until can't go any further. Now return to the starting position. Remember to keep the shoulders and the rest of the body still. Perform this three times.

3 


\section{Table 2 (on next page)}

Segment angle ROM mean and standard deviation.

ROM data (mean \pm SD) for motion capture (mocap) and inertial sensors (IMU) for 11 participants (5 for cervical flexion/extension). Segment angles are reported for all spinal segments (S1, L1, T6, T1 and SK). The movements from left to right were flexion, extension, lateral flexion, cervical rotation, and cervical flexion/extension. 
1 Table 2: Segment angle ROM mean and standard deviation. ROM data (mean \pm SD) for motion capture (mocap) and inertial 2 sensors (IMU) for 11 participants (5 for cervical flexion/extension). Segment angles are reported for all spinal segments (S1, L1,

$3 \mathrm{~T} 6, \mathrm{~T} 1$ and SK). The movements from left to right were flexion, extension, lateral flexion, cervical rotation, and cervical

4 flexion/extension.

$\begin{array}{cc}\text { S1 } & \text { mocap } \\ & \text { IMU } \\ \text { L1 } & \text { mocap } \\ & \text { IMU } \\ \text { T6 } & \text { mocap } \\ & \text { IMU } \\ \text { T1 } & \text { mocap } \\ & \text { IMU } \\ \text { SK } & \text { mocap }\end{array}$

\begin{tabular}{ccccc} 
Flex & Ext & Lat Flex & Cer Rot & Cer F/E \\
\hline \hline $62.3 \pm 13.9^{\circ}$ & $9.6 \pm 6.0^{\circ}$ & $7.6 \pm 2.2^{\circ}$ & - & - \\
$60.5 \pm 13.7^{\circ}$ & $9.4 \pm 5.8^{\circ}$ & $7.6 \pm 2.3^{\circ}$ & - & - \\
\hline $110.8 \pm 13.3^{\circ}$ & $24.7 \pm 11.1^{\circ}$ & $31.2 \pm 5.2^{\circ}$ & - & - \\
$111.0 \pm 13.2^{\circ}$ & $24.8 \pm 11.3^{\circ}$ & $30.9 \pm 4.9^{\circ}$ & - & - \\
\hline $125.4 \pm 11.2^{\circ}$ & $32.5 \pm 10.4^{\circ}$ & $45.9 \pm 7.6^{\circ}$ & - & - \\
$125.5 \pm 11.0^{\circ}$ & $32.4 \pm 10.5^{\circ}$ & $47.3 \pm 7.9^{\circ}$ & - & - \\
\hline $107.4 \pm 11.2^{\circ}$ & $43.4 \pm 9.7^{\circ}$ & $52.1 \pm 9.9^{\circ}$ & - & - \\
$107.9 \pm 11.3^{\circ}$ & $43.7 \pm 9.8^{\circ}$ & $53.8 \pm 10.1^{\circ}$ & - & - \\
\hline- & - & - & $73.5 \pm 10.2^{\circ}$ & $123.7 \pm 14.9^{\circ}$ \\
- & - & - & $73.4 \pm 10.1^{\circ}$ & $124.1 \pm 14.2^{\circ}$
\end{tabular}

5 


\section{Table 3 (on next page)}

Joint angle ROM mean and standard deviation

ROM data (mean \pm SD) for motion capture (mocap) and inertial sensors (IMU) for 11 participants (5 for cervical flexion/extension). Joint angles are reported for lumbar, lower thoracic, upper thoracic, cervical, and an overall mean. The movements from left to right were flexion, extension, lateral flexion, cervical rotation, and cervical flexion/extension. 
1 Table 3: Joint angle ROM mean and standard deviation. ROM data (mean \pm SD) for motion capture (mocap) and inertial 2 sensors (IMU) for 11 participants (5 for cervical flexion/extension). Joint angles are reported for lumbar, lower thoracic, upper 3 thoracic, cervical, and an overall mean. The movements from left to right were flexion, extension, lateral flexion, cervical 4 rotation, and cervical flexion/extension.

\begin{tabular}{|c|c|c|c|c|c|c|}
\hline & & Flex & Ext & Lat Flex & Cer Rot & Cer F/E \\
\hline \multirow{2}{*}{\multicolumn{2}{|c|}{ mocap }} & $\bar{c} 50.5 \pm 10.3^{\circ}$ & $18.8 \pm 6.8^{\circ}$ & $26.1 \pm 4.2^{\circ}$ & - & - \\
\hline & & $51.7 \pm 10.0^{\circ}$ & $19.1 \pm 7.1^{\circ}$ & $25.8 \pm 4.4^{\circ}$ & - & - \\
\hline 눈 & \multirow{2}{*}{$\begin{array}{l}\text { mocap } \\
\text { IMU }\end{array}$} & $15.6 \pm 6.9^{\circ}$ & $9.0 \pm 4.8^{\circ}$ & $14.1 \pm 5.6^{\circ}$ & - & - \\
\hline & & $16.1 \pm 6.9^{\circ}$ & $9.0 \pm 4.7^{\circ}$ & $14.4 \pm 5.3^{\circ}$ & - & - \\
\hline 当 & \multirow{2}{*}{$\begin{array}{l}\text { mocap } \\
\text { IMU }\end{array}$} & $20.6 \pm 8.7^{\circ}$ & $11.9 \pm 6.0^{\circ}$ & $5.3 \pm 4.4^{\circ}$ & - & - \\
\hline & & $20.6 \pm 8.6^{\circ}$ & $12.4 \pm 5.8^{\circ}$ & $6.0 \pm 4.0^{\circ}$ & - & - \\
\hline & \multirow{2}{*}{$\begin{array}{l}\text { mocap } \\
\text { IMU }\end{array}$} & - & - & - & $67.8 \pm 9.3^{\circ}$ & $93.5 \pm 11.3^{\circ}$ \\
\hline 3 & & - & - & - & $68.1 \pm 9.0^{\circ}$ & $93.7 \pm 10.7^{\circ}$ \\
\hline
\end{tabular}




\section{Table 4 (on next page)}

Segment angle analysis for ROM and kinematics

ROM data: nonparametric Bland-Altman median bias and limits of agreement (1.45 IQR).

Kinematics data: mean and standard deviation of root mean square error (RMSE), maximum absolute error (MAE) and Spearman correlation coefficient $(\rho)$. Segment angles are reported for all spinal segments (S1, L1, T6, T1 and SK) and an overall mean. Reliability Analysis (RA): intraclass correlation coefficient (ICC) with lower and upper bounds (LB and UB), standardised error measurement (SEM), and minimum detectable change (MDC). The movements from left to right were flexion, extension, lateral flexion, cervical rotation, and cervical flexion/extension. 
1 Table 4: Segment angle analysis for ROM and kinematics. ROM data: nonparametric Bland-Altman median bias and limits of agreement (1.45 IQR). Kinematics data: mean and standard deviation of root mean square error (RMSE), maximum absolute error (MAE) and Spearman correlation coefficient $(\rho)$. Segment angles are reported for all spinal segments (S1, L1, T6, T1 and SK) and an overall mean. Reliability Analysis (RA): intraclass correlation coefficient (ICC) with lower and upper bounds (LB and UB), standardised error measurement (SEM), and minimum detectable change (MDC). The movements from left to right were flexion, extension, lateral flexion, cervical rotation, and cervical flexion/extension.

\begin{tabular}{|c|c|c|c|c|c|c|}
\hline & & Flex & Ext & Lat Flex & Cer Rot & Cer F/E \\
\hline \multirow{5}{*}{ S1 } & bias \pm LOA & $-0.9 \pm 1.2^{\circ}$ & $-0.1 \pm 0.7^{\circ}$ & $0.0 \pm 0.6^{\circ}$ & - & - \\
\hline & RMSE & $1.0 \pm 0.4^{\circ}$ & $0.9 \pm 0.7^{\circ}$ & $0.3 \pm 0.2^{\circ}$ & - & - \\
\hline & MAE & $2.3 \pm 0.7^{\circ}$ & $2.0 \pm 1.0^{\circ}$ & $1.1 \pm 0.9^{\circ}$ & - & - \\
\hline & $\rho$ & $0.99 \pm 0.01$ & $0.98 \pm 0.02$ & $0.99 \pm 0.01$ & - & - \\
\hline & bias \pm LOA & $0.2 \pm 1.7^{\circ}$ & $0.0 \pm 1.5^{\circ}$ & $-0.2 \pm 1.3^{\circ}$ & - & - \\
\hline \multirow{3}{*}{ L1 } & RMSE & $2.0 \pm 0.4^{\circ}$ & $1.6 \pm 0.6^{\circ}$ & $1.0 \pm 0.3^{\circ}$ & - & - \\
\hline & MAE & $4.7 \pm 0.7^{\circ}$ & $2.8 \pm 0.9^{\circ}$ & $2.5 \pm 0.9^{\circ}$ & - & - \\
\hline & $\rho$ & $0.99 \pm 0.01$ & $0.99 \pm 0.01$ & $0.99 \pm 0.01$ & - & - \\
\hline \multirow{4}{*}{ T6 } & bias \pm LOA & $0.1 \pm 1.4^{\circ}$ & $0.0 \pm 1.0^{\circ}$ & $1.1 \pm 2.3^{\circ}$ & - & - \\
\hline & RMSE & $2.5 \pm 0.6^{\circ}$ & $2.5 \pm 0.8^{\circ}$ & $1.7 \pm 0.5^{\circ}$ & - & - \\
\hline & MAE & $5.4 \pm 1.0^{\circ}$ & $3.6 \pm 1.0^{\circ}$ & $4.3 \pm 1.3^{\circ}$ & - & - \\
\hline & $\rho$ & $0.99 \pm 0.01$ & $0.99 \pm 0.01$ & $0.99 \pm 0.01$ & - & - \\
\hline \multirow{4}{*}{ T1 } & bias \pm LOA & $0.4 \pm 1.2^{\circ}$ & $0.3 \pm 1.0^{\circ}$ & $1.7 \pm 2.0^{\circ}$ & - & - \\
\hline & RMSE & $2.1 \pm 0.4^{\circ}$ & $1.3 \pm 0.3^{\circ}$ & $1.8 \pm 0.4^{\circ}$ & - & - \\
\hline & MAE & $5.3 \pm 1.2^{\circ}$ & $3.0 \pm 0.7^{\circ}$ & $4.4 \pm 0.8^{\circ}$ & - & - \\
\hline & $\rho$ & $0.99 \pm 0.01$ & $0.99 \pm 0.01$ & $0.99 \pm 0.01$ & - & - \\
\hline \multirow{4}{*}{ SK } & bias \pm LOA & - & - & - & $-0.5 \pm 2.3^{\circ}$ & $0.5 \pm 2.5^{\circ}$ \\
\hline & RMSE & - & - & - & $1.2 \pm 0.3^{\circ}$ & $1.7 \pm 0.3^{\circ}$ \\
\hline & MAE & - & - & - & $3.0 \pm 0.5^{\circ}$ & $5.0 \pm 1.1^{\circ}$ \\
\hline & $\rho$ & - & - & - & $0.92 \pm 0.09$ & $0.99 \pm 0.01$ \\
\hline \multirow{4}{*}{ Mean } & bias \pm LOA & $0.1 \pm 1.8^{\circ}$ & $0.0 \pm 1.1^{\circ}$ & $0.5 \pm 2.4^{\circ}$ & $-0.5 \pm 2.3^{\circ}$ & $0.5 \pm 2.5^{\circ}$ \\
\hline & RMSE & $1.9 \pm 0.7^{\circ}$ & $1.6 \pm 0.8^{\circ}$ & $1.2 \pm 0.7^{\circ}$ & $1.2 \pm 0.3^{\circ}$ & $1.7 \pm 0.3^{\circ}$ \\
\hline & MAE & $4.4 \pm 1.6^{\circ}$ & $2.8 \pm 1.1^{\circ}$ & $3.0 \pm 1.7^{\circ}$ & $3.0 \pm 0.5^{\circ}$ & $5.0 \pm 1.1^{\circ}$ \\
\hline & $\rho$ & $0.99 \pm 0.01$ & $0.99 \pm 0.01$ & $0.99 \pm 0.01$ & $0.92 \pm 0.09$ & $0.99 \pm 0.01$ \\
\hline \multirow{4}{*}{$\mathbf{R A}$} & ICC & 0.999 & 0.999 & 0.996 & 0.990 & 0.997 \\
\hline & ICC(LB UB) & $(0.9990 .999)$ & (0.998 0.999$)$ & (0.996 0.997$)$ & $\left(\begin{array}{lll}0.986 & 0.992)\end{array}\right.$ & (0.992 0.999$)$ \\
\hline & SEM & $0.6^{\circ}$ & $0.5^{\circ}$ & $1.1^{\circ}$ & $1.0^{\circ}$ & $0.75^{\circ}$ \\
\hline & MDC & $1.6^{\circ}$ & $1.3^{\circ}$ & $2.9^{\circ}$ & $2.7^{\circ}$ & $2.1^{\circ}$ \\
\hline
\end{tabular}




\section{Table 5 (on next page)}

Joint angle analysis for ROM and kinematics

ROM data: nonparametric Bland-Altman median bias and limits of agreement (1.45 IQR). Kinematics data: mean and standard deviation of root mean square error (RMSE), maximum absolute error (MAE) and Spearman correlation coefficient ( $\rho)$. Joint angles are reported for lumbar, lower thoracic, upper thoracic, cervical, and an overall mean. Reliability Analysis: intraclass correlation coefficient (ICC), standardised error measurement (SEM), and minimum detectable change (MDC). The movements from left to right were flexion, extension, lateral flexion, cervical rotation, and cervical flexion/extension. 
1 Table 5: Joint angle analysis for ROM and kinematics. ROM data: nonparametric Bland-Altman median bias and limits of agreement (1.45 IQR). Kinematics data: mean and standard deviation of root mean square error (RMSE), maximum absolute error (MAE) and Spearman correlation coefficient $(\rho)$. Joint angles are reported for lumbar, lower thoracic, upper thoracic, cervical, and an overall mean. Reliability Analysis: intraclass correlation coefficient (ICC), standardised error measurement (SEM), and minimum detectable change (MDC). The movements from left to right were flexion, extension, lateral flexion, 6 cervical rotation, and cervical flexion/extension.

\begin{tabular}{|c|c|c|c|c|c|c|}
\hline \multirow{2}{*}{ 쳘 } & 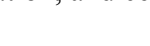 & Flex & Ext & Lat Flex & Cer Rot & $\operatorname{Cer} \mathbf{F} / \mathbf{E}$ \\
\hline & bias \pm LOA & $1.0 \pm 1.7^{\circ}$ & $0.3 \pm 1.6^{\circ}$ & $-1.0 \pm 6.4^{\circ}$ & 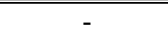 & - \\
\hline \multirow{2}{*}{ 突 } & RMSE & $1.9 \pm 0.4^{\circ}$ & $1.4 \pm 0.7^{\circ}$ & $2.4 \pm 1.0^{\circ}$ & - & - \\
\hline & MAE & $4.4 \pm 1.0^{\circ}$ & $2.9 \pm 1.6^{\circ}$ & $5.4 \pm 1.8^{\circ}$ & - & - \\
\hline & bias $\pm \mathrm{LOA}$ & $0.3 \pm 1.5^{\circ}$ & $0.1 \pm 1.2^{\circ}$ & $0.3 \pm 3.6^{\circ}$ & - & - \\
\hline & RMSE & $1.3 \pm 0.6^{\circ}$ & $1.4 \pm 0.5^{\circ}$ & $1.5 \pm 0.5^{\circ}$ & - & - \\
\hline & bias \pm LOA & $0.0 \pm 1.9^{\circ}$ & $0.6 \pm 1.3^{\circ}$ & $0.8 \pm 5.6^{\circ}$ & - & - \\
\hline & RMSE & $2.7 \pm 1.2^{\circ}$ & $2.8 \pm 1.3^{\circ}$ & $2.9 \pm 1.3^{\circ}$ & - & - \\
\hline & MAE & $4.6 \pm 1.3^{\circ}$ & $4.2 \pm 1.6^{\circ}$ & $6.5 \pm 2.2^{\circ}$ & - & - \\
\hline & $\rho$ & $0.98 \pm 0.01$ & $0.98 \pm 0.03$ & $0.48 \pm 0.53$ & - & - \\
\hline$\theta$ & bias \pm LOA & - & - & - & $0.0 \pm 2.3^{\circ}$ & $0.4 \pm 1.9^{\circ}$ \\
\hline \multirow{4}{*}{ Mean } & bias \pm LOA & $0.5 \pm 1.7^{\circ}$ & $0.3 \pm 1.5^{\circ}$ & $0.2 \pm 4.8^{\circ}$ & $0.0 \pm 2.3^{\circ}$ & $0.4 \pm 1.9^{\circ}$ \\
\hline & RMSE & $2.0 \pm 1.0^{\circ}$ & $1.8 \pm 1.1^{\circ}$ & $2.3 \pm 1.1^{\circ}$ & $1.7 \pm 0.8^{\circ}$ & $2.6 \pm 0.9^{\circ}$ \\
\hline & MAE & $3.9 \pm 1.3^{\circ}$ & $3.3 \pm 1.6^{\circ}$ & $5.2 \pm 2.0^{\circ}$ & $3.7 \pm 1.2^{\circ}$ & $6.1 \pm 1.0^{\circ}$ \\
\hline & $\rho$ & $0.99 \pm 0.02$ & $0.98 \pm 0.03$ & $0.82 \pm 0.38$ & $0.92 \pm 0.09$ & $0.99 \pm 0.01$ \\
\hline \multirow{4}{*}{$\mathbf{R A}$} & $\mathrm{ICC}$ & 0.998 & 0.989 & 0.952 & 0.986 & 0.993 \\
\hline & ICC(LB UB) & (0.997 0.998$)$ & $\left(\begin{array}{lll}0.985 & 0.992)\end{array}\right.$ & $\left(\begin{array}{lll}0.942 & 0.961)\end{array}\right.$ & $\left(\begin{array}{lll}0.981 & 0.990\end{array}\right)$ & $\left(\begin{array}{ll}0.981 & 0.998\end{array}\right)$ \\
\hline & SEM & $0.8^{\circ}$ & $0.7^{\circ}$ & $2.1^{\circ}$ & $1.1^{\circ}$ & $0.9^{\circ}$ \\
\hline & $\mathrm{MDC}$ & $2.1^{\circ}$ & $2.0^{\circ}$ & $5.7^{\circ}$ & $2.9^{\circ}$ & $2.4^{\circ}$ \\
\hline
\end{tabular}




\section{Table 6(on next page)}

Kolmogorov-Smirnov normality test

$\alpha$ values for sagittal, frontal and transversal planes, for both segment and joint angles. Values who passed the test in bold $(\alpha>0.05)$. 
1 Table 6: Kolmogorov-Smirnov normality test. $\alpha$ values for sagittal, frontal and transversal planes, for both segment and joint 2 angles. Values who passed the test in bold $(\alpha>0.05)$.

\begin{tabular}{lccc} 
& Sagittal & Frontal & Transversal \\
\cline { 2 - 4 } Segment & $\mathbf{0 . 7 5}$ & $<0.01$ & $<0.01$ \\
Joint & $\mathbf{0 . 1 9}$ & $\mathbf{0 . 2 0}$ & $<0.01$
\end{tabular}

3 


\section{Figure 1}

Sensors and cluster placement and reference system description.

(A) Sensor and cluster with their reference system. (B) Example of all sensors and clusters mounted. The spinal segments (lumbar, low thoracic, upper thoracic and cervical) are defined by sensors pairs (S1-L1, L1-T6, T6-T1, and T1-SK) 


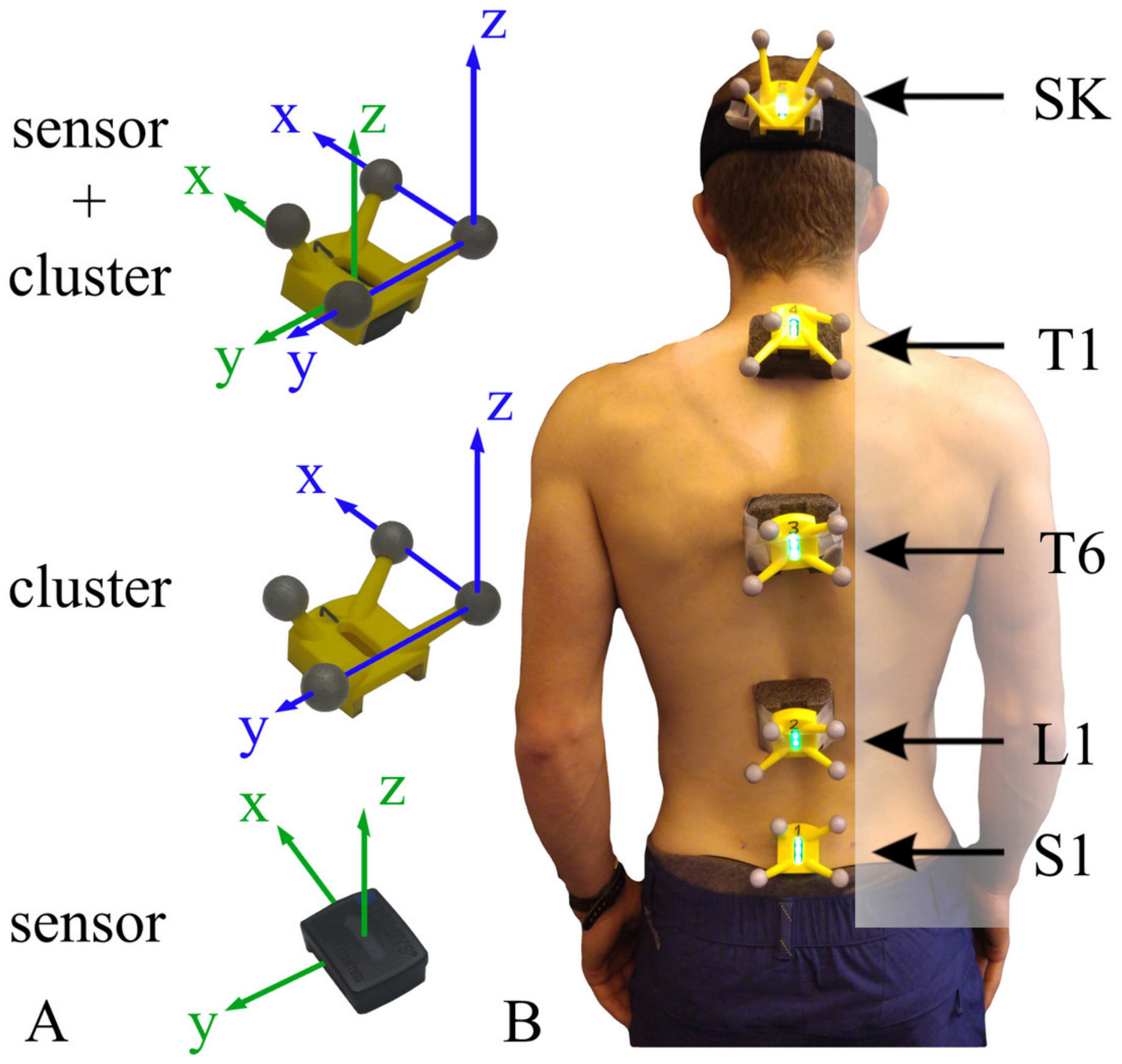


Figure 2

Description of the functional movements included in the protocol.

Movement included in the protocol: flexion (A), extension (B), lateral flexion (C), cervical rotation (D) and cervical flexion/extension (E).
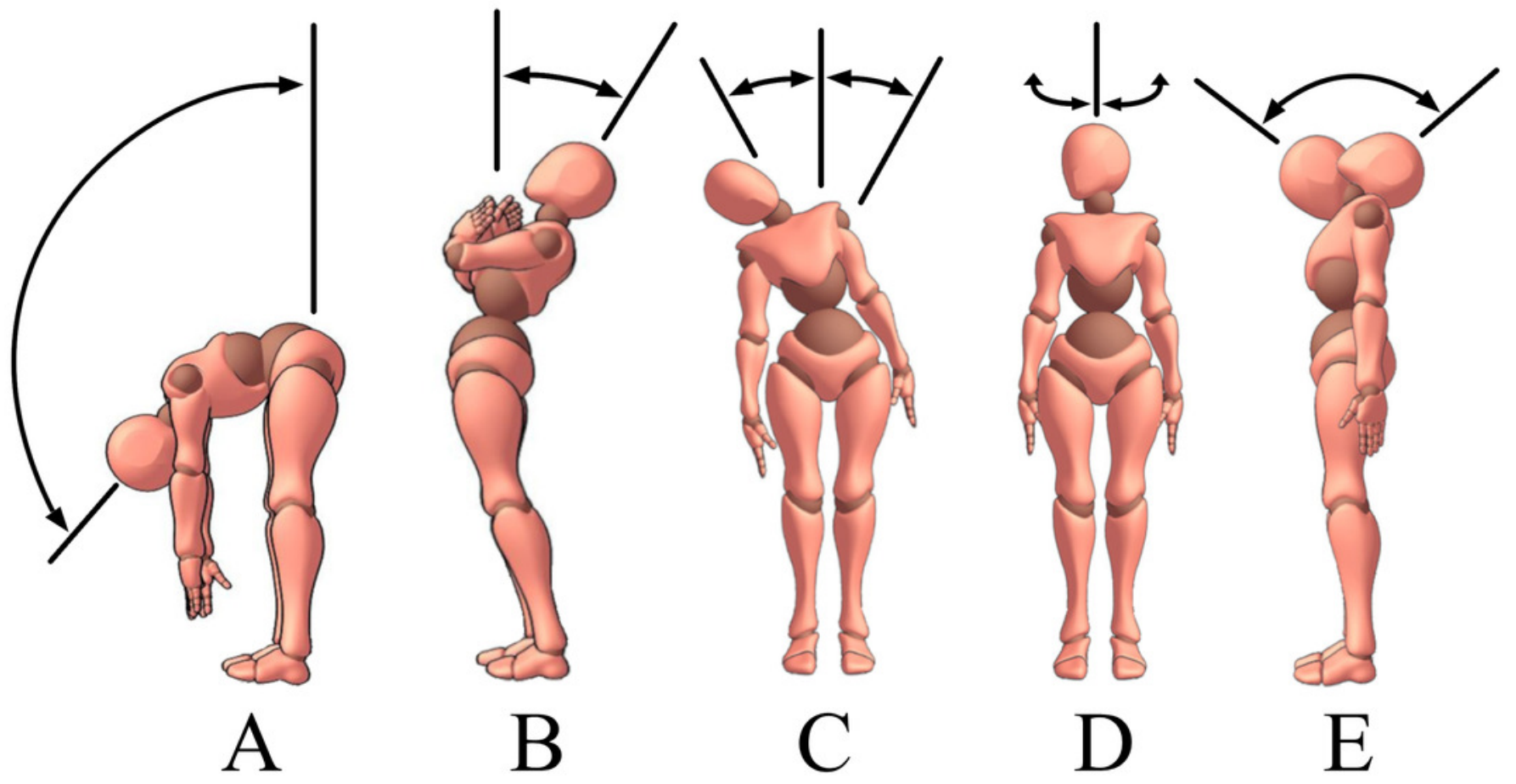
Figure 3

Explanation of the kinematic constraints and rotations between different reference systems.

(A) Representation of sensor axes and kinematic constraints axes. The sensor local reference system is shown in green, the kinematic constraints axes are shown in red, and the global reference system planes are shown in black. (B) Segment angle $\left({ }_{G}^{S} q_{1}\right)$ and a joint angle $\left({ }_{S}^{S}\right.$ $\left.q_{2-1}\right)$ orientation. The sensor angle orientation $\left({ }_{F}^{S} q_{1}\right)$ with respect to the fixed reference system (blue), the fixed reference system (orange) orientation $\left({ }_{G}^{F} q_{1}\right)$ with respect to the global reference system, and body reference system orientation with respect to the sensor reference system $\left({ }_{S}^{B} q_{2}\right)$ are shown.
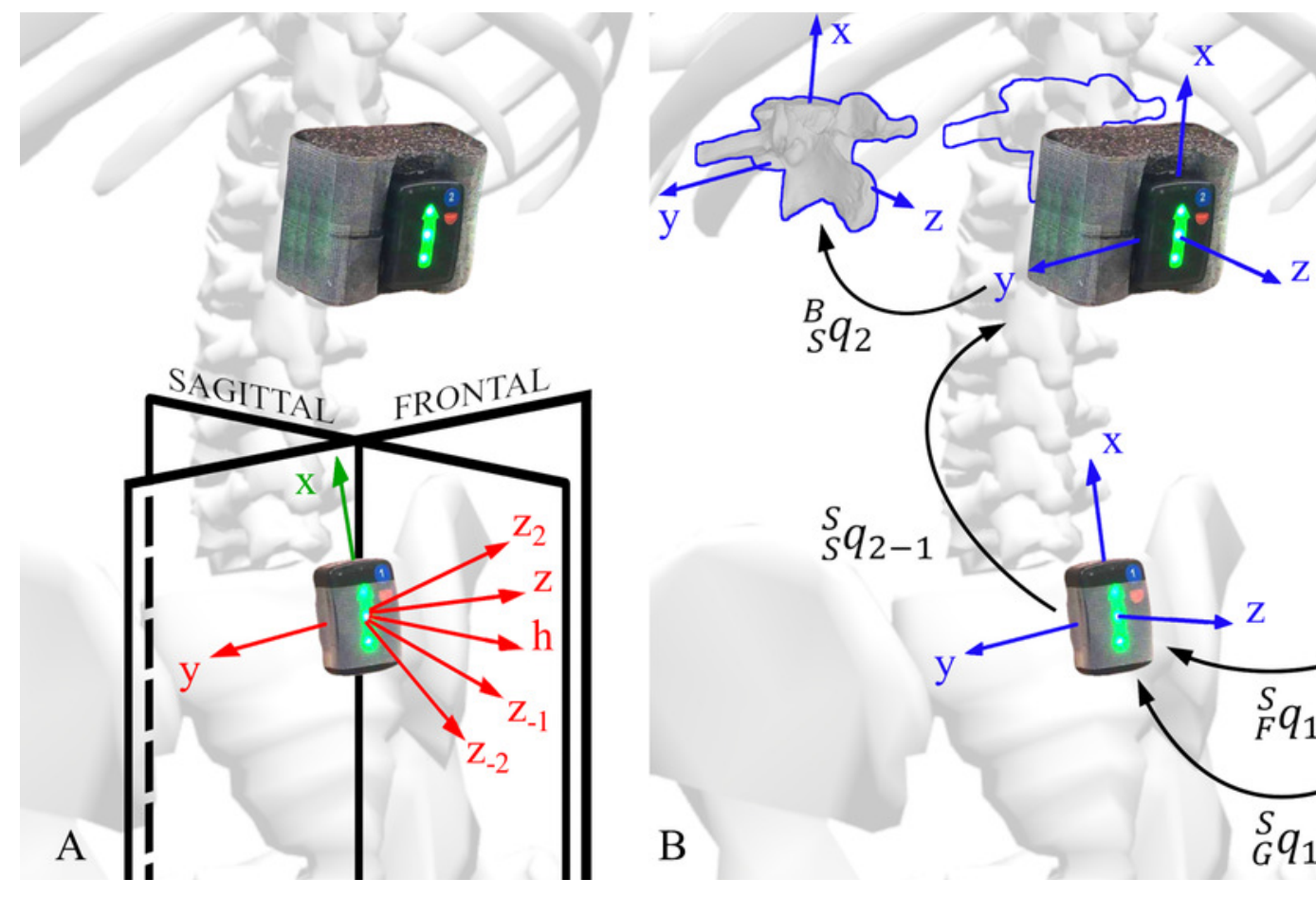

Fixed

reference

system

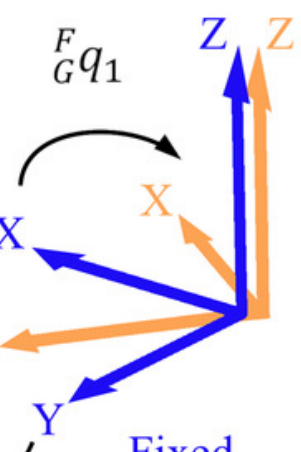

Fixed reference system

B ${ }_{G}^{S} q_{1}$ 
Figure 4

Clinical angles description and example of the segmentation process.

Clinical angles: flexion/extension angle in red, lateral flexion angle in green, axial rotation angle in blue. (B) Example of joint angle segmentation for lumbar segment during flexion, (C) lower thoracic segment during lateral flexion, (D) cervical segment during cervical flexion/extension.

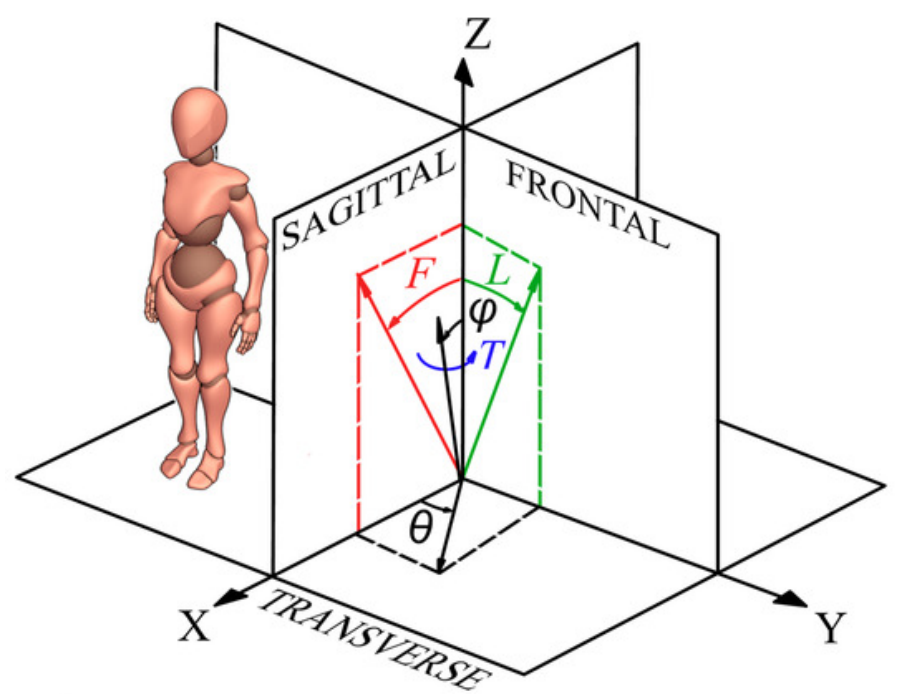

A

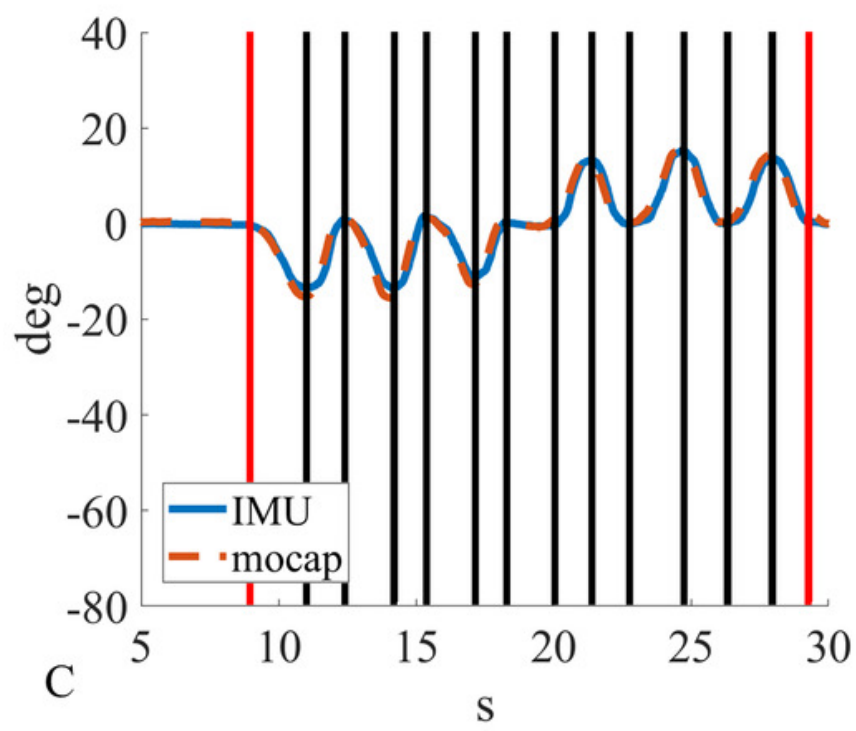

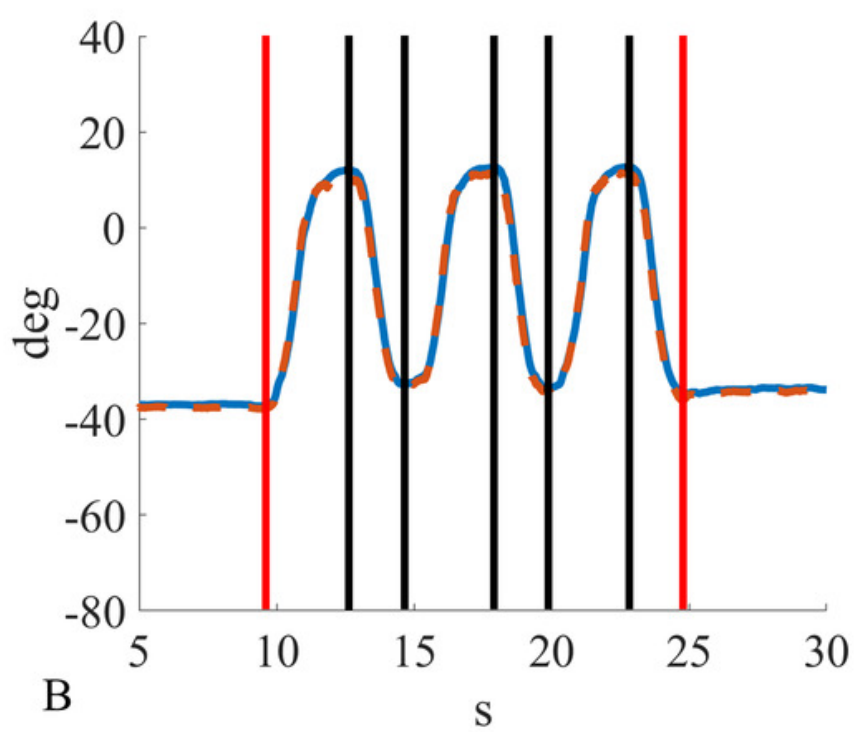

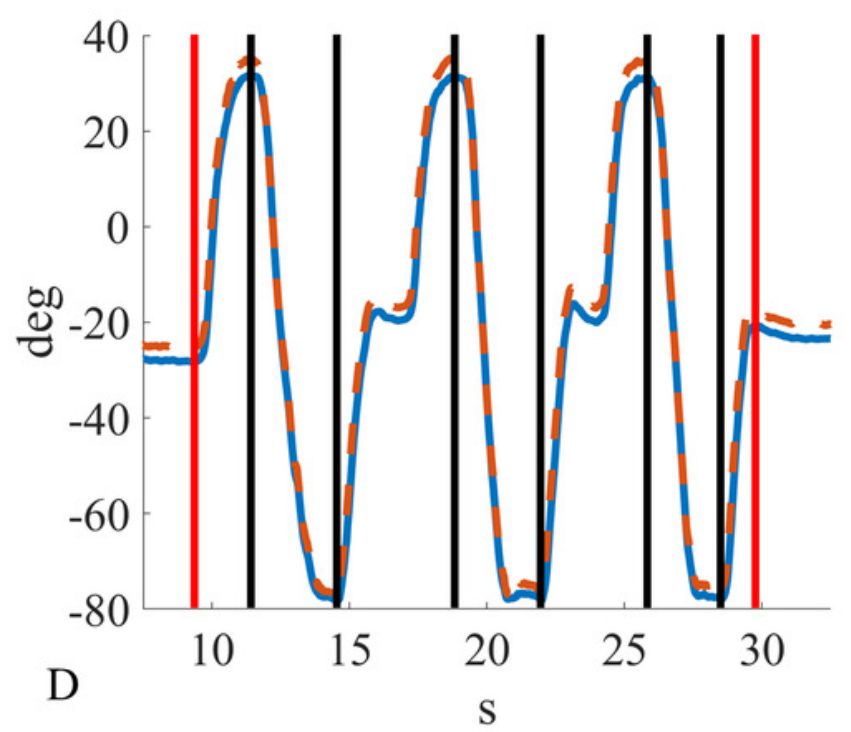




\section{Figure 5}

Bland-Altman plots.

Comparison of IMU to motion capture joint angles ROM for flexion (A), extension (B), lateral flexion (C), cervical rotation (D), cervical flexion/extension (E). Cervical rotation segment angles (F) were also added. A-C represent the lumbar segment (cyan), lower thoracic segment (black), and upper thoracic segment (green). D and E represent the cervical segment (blue). F represents the head sensor SK (blue). All the plots show: median bias (black line), limits of agreement (1.45 IQR, black dotted lines), and 10\% error threshold (red dotted lines).

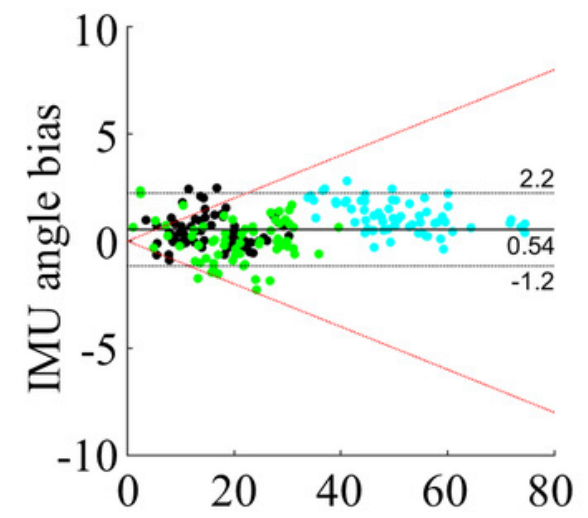

A

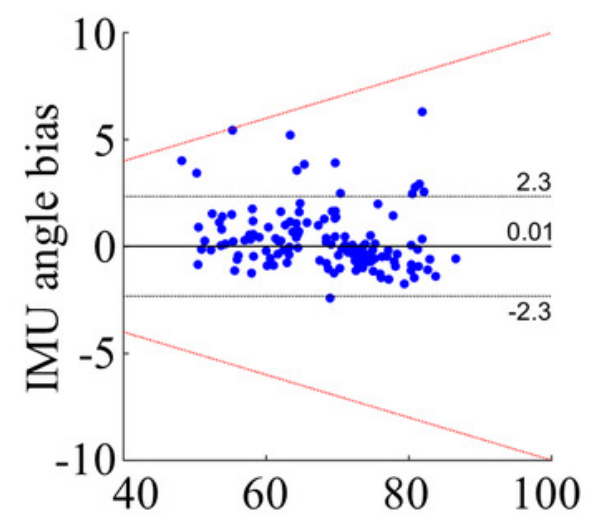

$\mathrm{D}$

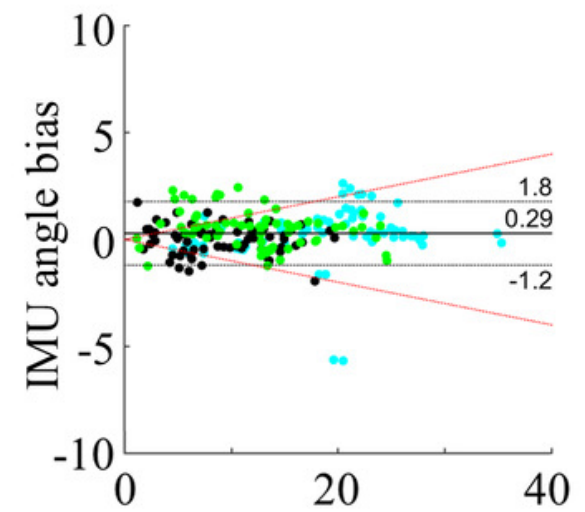

B

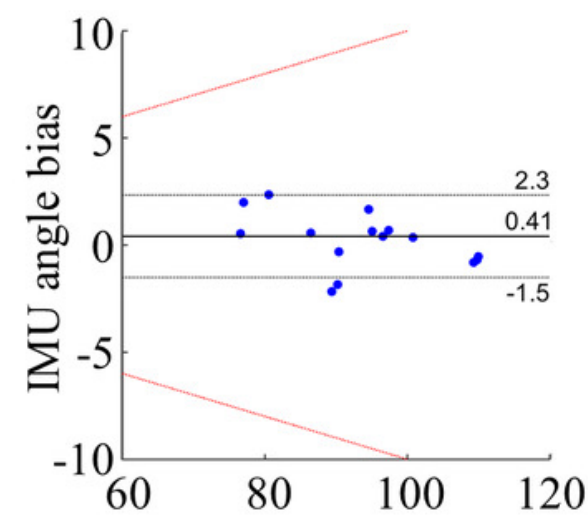

E
Mean angle

Mean angle
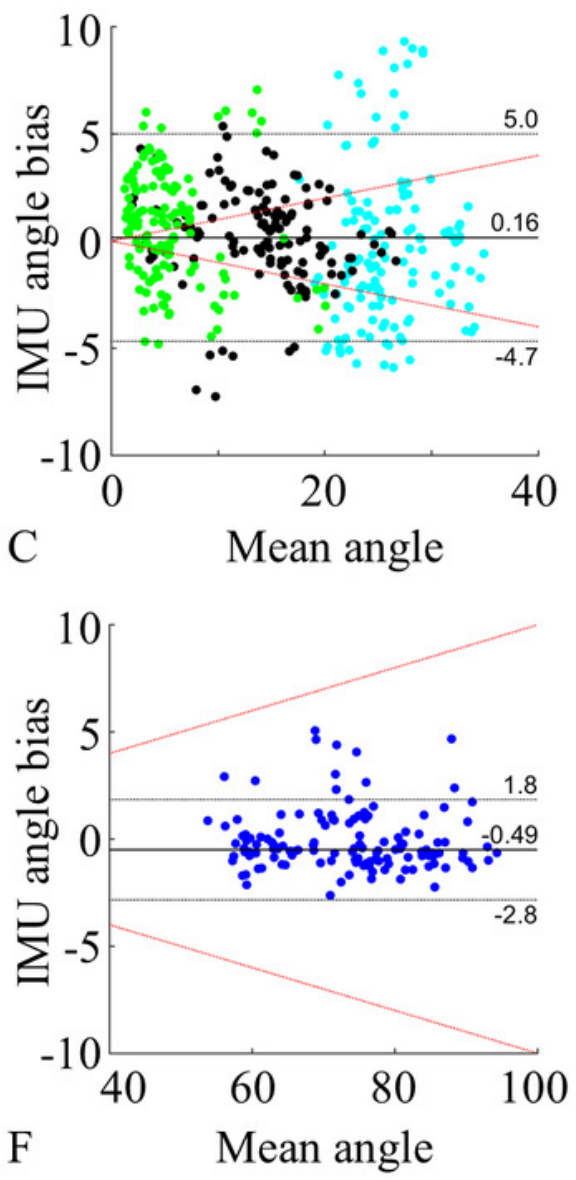


\section{Figure 6}

Percentage of frames error analysis.

Errors are divided into 4 error classes (err $<2^{\circ}, 2^{\circ}<$ err $<4^{\circ}, 4^{\circ}<\operatorname{err}<6^{\circ}$, err $>6^{\circ}$ ) for absolute errors ( $A$ and C), and 4 error classes (err $<10 \%, 10 \%<$ err $<20 \%, 20 \%<$ err $<$ $40 \%$, err $>40 \%$ ) for percent errors ( $B$ and $D$ ). The amount of frames recorded in a specific class, for a specific movement, are reported on the y axis as frequency observed expressed in percentage. All functional movements are represented. Segment angles errors are shown in $A$ and $B$ while joint angle errors are shown in $C$ and $D$. 

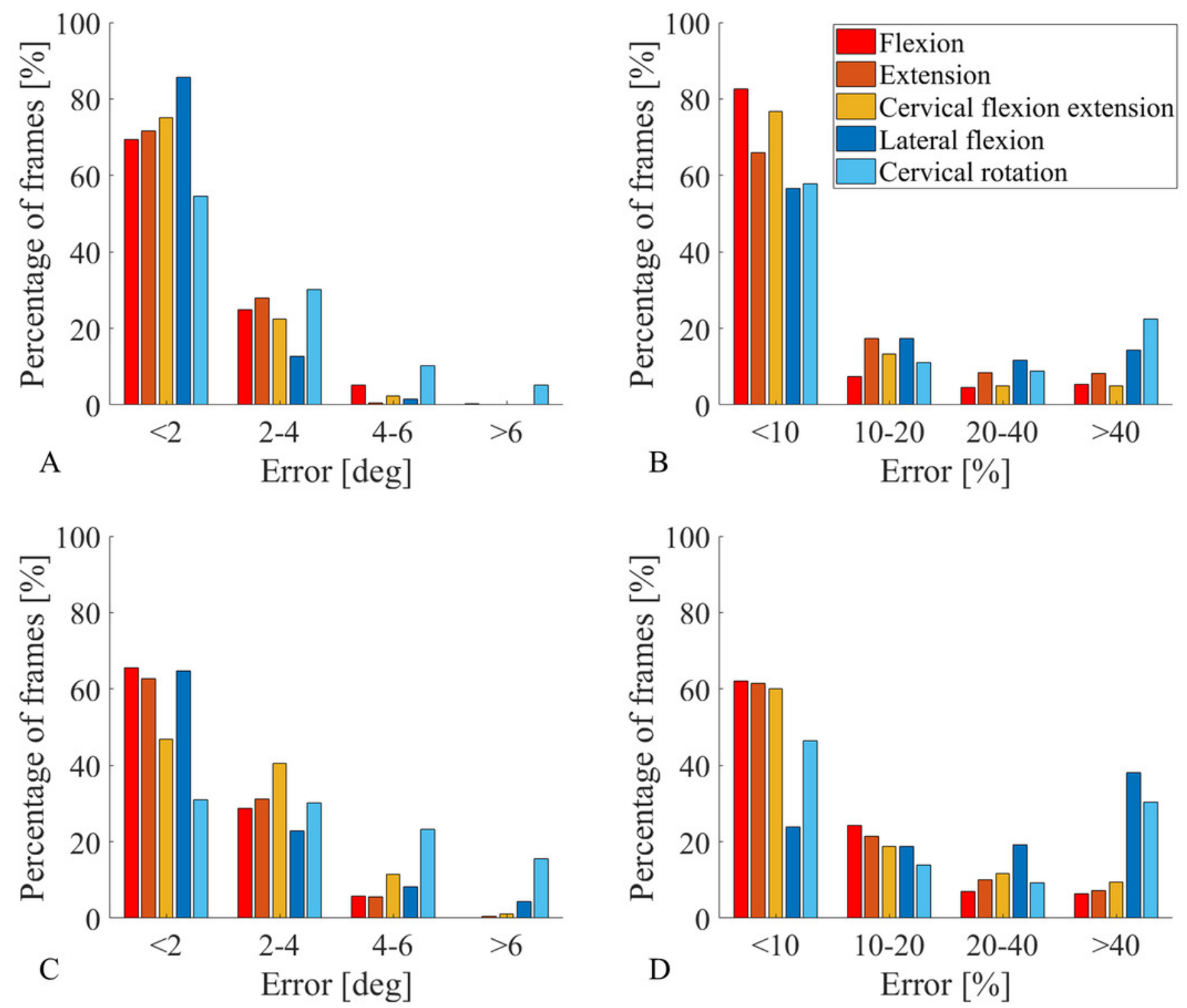
Figure 7

Constraint number optimisation

ROM measurement error absolute value, plotted against constraint number for all segment (A) and joint (B) angles. Mean (blue line) and standard deviation (orange circle) values are calculated across all participants, also the optimum value is shown (red diamond).
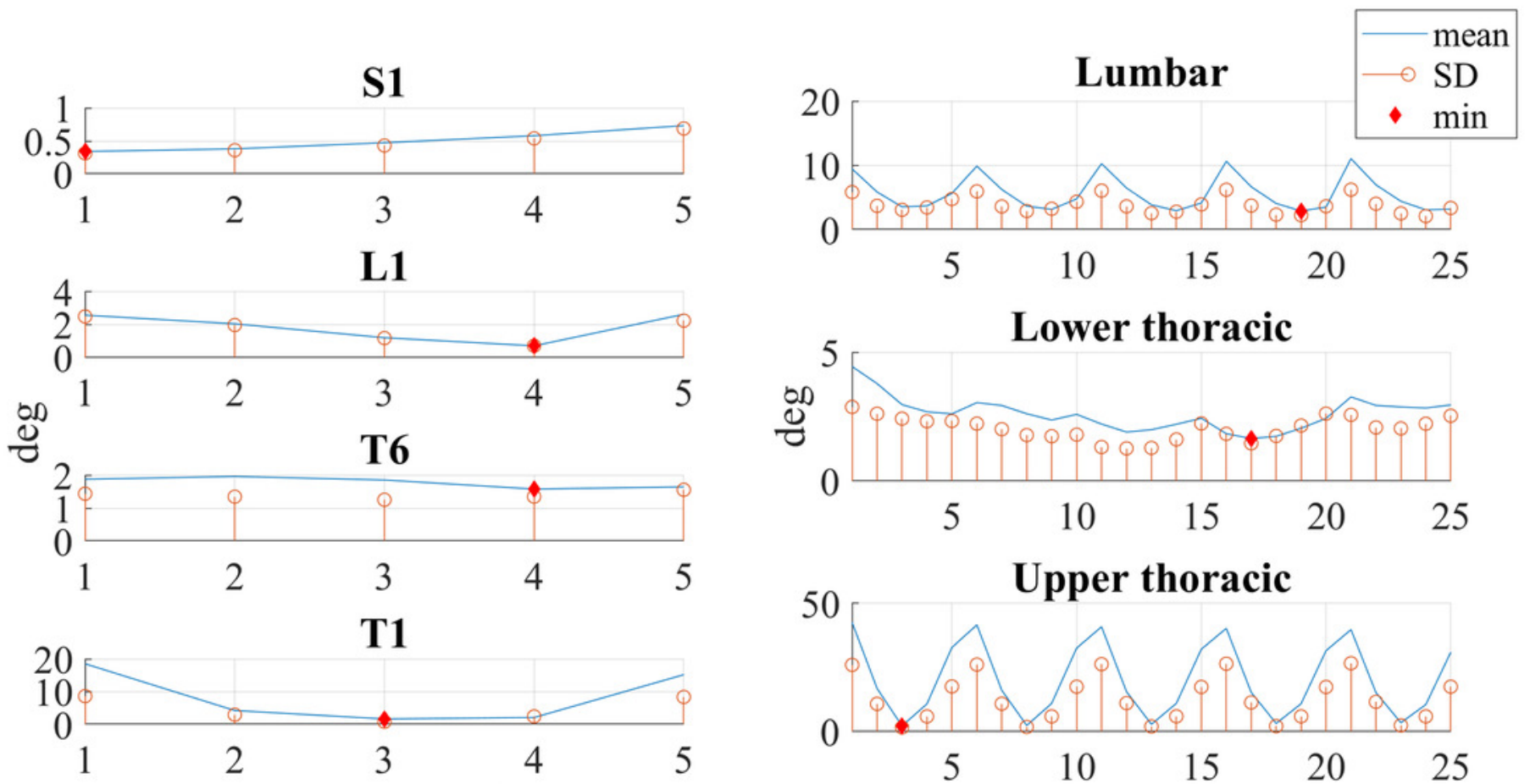

A

Constraint number
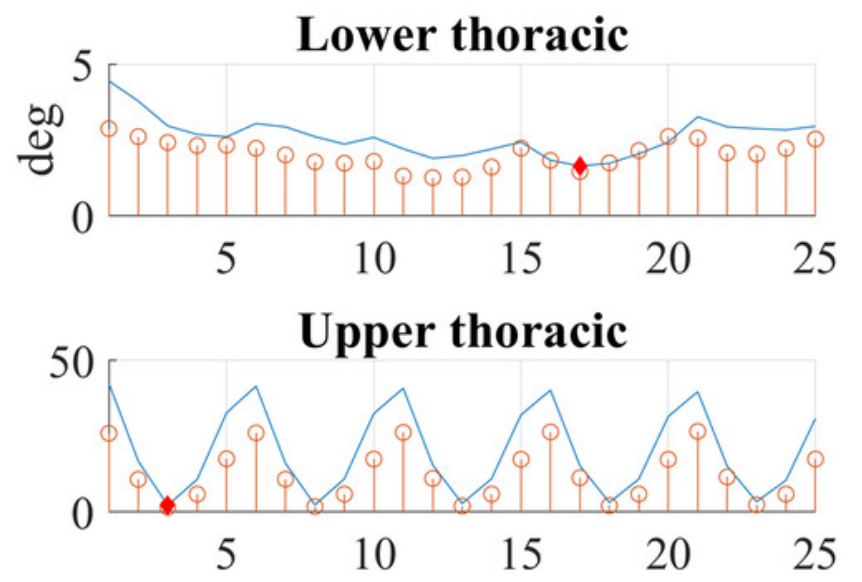

B

Constraint number 
Table 7(on next page)

Constraints number

Constraint number associated to constraint axes combinations for joint angles 
1 Table A1: Constraint number associated to constraint axes combinations for joint angles.

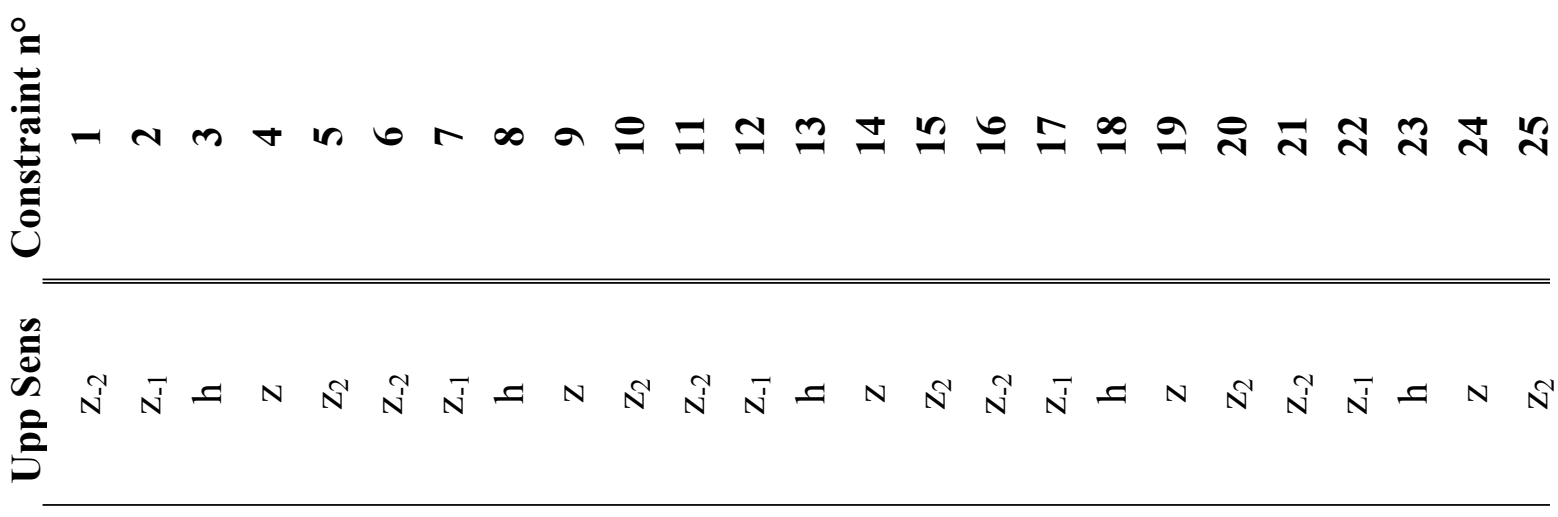

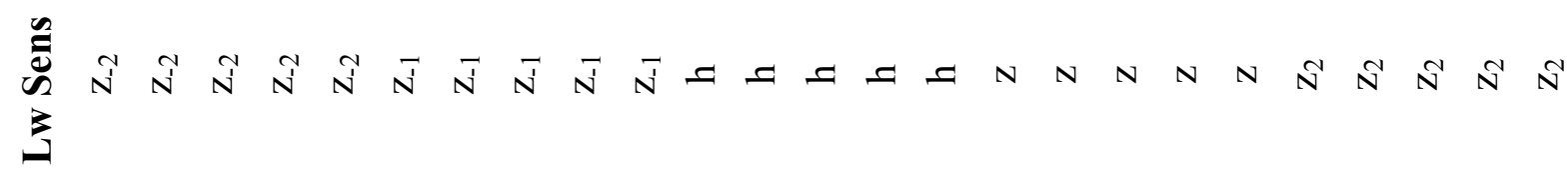




\section{Table 8 (on next page)}

Constraint axes optima for segment and joint angles.

Constraint number and associated axis are shown with mean and standard deviation of ROM measurement error absolute values. 
1 Table A2: Constraint axes optima for segment and joint angles. Constraint number and associated axis are shown with mean 2 and standard deviation of ROM measurement error absolute values.

\begin{tabular}{lccccccc} 
& \multicolumn{3}{c}{ Segment angles } & \multicolumn{3}{c}{ Joint angles } \\
\cline { 2 - 8 } Constraint $\mathbf{n}^{\circ}$ & S1 & L1 & T6 & T1 & Lumbar & Lw thoracic & Upp thoracic \\
\cline { 2 - 8 } & 1 & 4 & 4 & 3 & 19 & 17 & 3 \\
Constraint axis & $\hat{Z}_{-2}$ & $\hat{Z}$ & $\hat{Z}$ & $\hat{h}$ & $\hat{Z}-\hat{z}$ & $\hat{Z}-\hat{Z}_{-1}$ & $\hat{Z}_{-2^{-}} \hat{h}$ \\
Mean & $0.3^{\circ}$ & $0.7^{\circ}$ & $1.6^{\circ}$ & $1.7^{\circ}$ & $2.9^{\circ}$ & $1.6^{\circ}$ & $2.2^{\circ}$ \\
SD & $0.3^{\circ}$ & $0.7^{\circ}$ & $1.4^{\circ}$ & $0.9^{\circ}$ & $2.3^{\circ}$ & $1.5^{\circ}$ & $1.6^{\circ}$
\end{tabular}

3 